\title{
Solar Eclipses and the Surface Properties of Water
}

\author{
Elmar C. Fuchs ${ }^{1}$ (D) - Gerrit Oudakker ${ }^{1} \cdot$ Martin Justinek $^{2} \cdot$ Nigel Dyer $^{1,3}$. \\ Jakob Woisetschläger ${ }^{1,4} \cdot$ Kevin Godines $^{5} \cdot$ Matthias Mäder $^{6}$. Friedemann T. Freund ${ }^{7,8}$
}

Received: 29 May 2019 / Accepted: 11 September 2019 / Published online: 30 September 2019

(c) The Author(s) 2019

\begin{abstract}
During four solar eclipse events (two annular, one total and one partial) a correlation was observed between a change in water surface tension and the magnitude of the optical coverage. During one eclipse, evaporation experiments were carried out which showed a reduction in water evaporation at the same time as a rise in the surface tension. The changes did not occur on a day without a solar eclipse and are not correlated to changes in temperature, pressure, humidity of the environment. The effects are delayed by 20, 85, 30 and 37 min, respectively, compared to the maximum eclipse. Possible mechanisms responsible for this effect are presented, the most likely hypothesis being reduced water/muon interaction due to solar wind and cosmic radiation blocking during an eclipse. As an alternative hypotheses, we propose a novel neutrino/water interaction and overview of other, less likely mechanisms.
\end{abstract}

Keywords Solar eclipse $\cdot$ Surface tension $\cdot$ Water

Jakob Woisetschläger

jakob.woisetschlaeger@tugraz.at

Elmar C. Fuchs

elmar.fuchs@wetsus.nl

1 Wetsus, European Centre of Excellence for Sustainable Water Technology, Leeuwarden, The Netherlands

2 Justinek Engineering, Vienna, Austria

3 Coherent Water Systems, Royal Leamington Spa, UK

4 Institute for Thermal Turbomachinery and Machine Dynamics, Graz University of Technology, Graz, Austria

5 Department of Bioengineering, Berkeley, UC, USA

6 Department of Geoscience \& Engineering, TU Delft, Delft, The Netherlands

7 NASA Earth Science Division, NASA Ames Research Center, Moffett Field, CA, USA

8 SETI Institute, Carl Sagan Center, Mountain View, CA, USA 


\section{Introduction}

Solar eclipses have fascinated humankind since the beginning of recorded history. As valuable resource for historians they allow certain historical events to be dated precisely thereby improving the accuracy of historical chronology. For example a solar eclipse of June 15 in 763 BC mentioned in an Assyrian eponym list (Rawlinson 1867) is an important timestamp for the chronology of the Ancient Orient. In pre-scientific times, before chemistry separated from alchemy, solar eclipses (aka "chemical weddings") were thought to be special insofar that certain (al-)chemical reactions would only take place during such events (Huang 2014). In the beginning of the 20th century some anecdotal evidence (Kolisko 1927) suggested a possible correlation between a solar eclipse and the capillary forces of an aqueous gold colloid solution; in 1975 investigations by Schütz and Schütz (1975) suggested a correlation between a solar eclipse and the mixing dynamics of water and glycerol. It should be mentioned that the solar eclipse was not visible at the location where the experiments took place, though. According to the knowledge of the authors none of the above mentioned experiments satisfy the standards of modern science and should therefore not be considered as scientific literature. However, they can serve as inspiration to explore actual effects that occur during a solar eclipse next to the obvious changes in illumination, temperature and weather conditions. Apart from changes to water physics, various experiments have been performed in order to detect anomalous gravitational behavior during solar eclipses. Some observations gave positive results and some failed to detect any noticeable effect. Allais reported in 1954 and in 1959 that the plane and/or amplitude of oscillation of a pendulum shifted during an eclipse (Allais 1975, 1999); however, an investigation by Slichter et al. (1965) failed to report any gravitational effect. In Saxl and Allen (1970) again confirmed the observations of Allais (1970), whereas in 1990 Kuusela confirmed the conclusion of Slichter reporting that no effect could be observed (Kuusela 1991). In Van Flandern and Yang (2003) provided a possible explanation for these effects. Rapid air movement for the bulk of the atmosphere above normal cloud levels are said to create a rise of atmospheric pressure causing the anomalies. The topic of gravitational effects during solar eclipses therefore seems to be explained as barometric variation effect.

\subsection{Effects on Earth's Ozone Layer}

There are several studies claiming that a solar eclipse has an impact on Earth's ozone layer (Bezverkhny et al. 1956; Fournier 1956; Kawabata1936; Khrigian et al. 1961; Jerlov et al. 1954; Pariisky and Chen-Chao 1958; Steblova 1961; Stranz 1961; Svensson 1968; Chakrabarty et al. 1997; Zerefos et al. 1999). These claims are based upon measurements of the amount of UV-B radiation on the surface during the eclipse, and they vary between a $40 \%$ decrease (Jerlov et al. 1954) to a 20-30\% increase (Khrigian et al. 1961; Steblova 1961) and even an unlikely increase of 300-400\% (Bezverkhny et al. 1956). However, comparable to the gravitational study situation, there are papers rejecting any relation between the solar eclipse and the ozone layer. Initially in Bojkov (1968) and subsequently in Blumthaler et al. (2006) showed convincingly that the differences observed can be explained by limb darkening (Bojkov 1968; Blumthaler et al. 2006), an effect that deals with the difference in spectral composition of radiation coming from either the limb or the center of the solar disk. Therefore, any secondary effects of a solar eclipse based upon changes in the ozone layer should be treated with caution. Nevertheless, some of these investigation should be mentioned here, for example 
the study by Chakrabarty et al. (1997) of the fluctuation in ozone column over Ahmedabad during the solar eclipse of 24 October 1995, and the study by Mims and Mims about the same topic during the solar eclipse of July 11th, 1991 (Mims and Mims 1993). Whereas the interpretation of their results might be disputable, their measurements show effects with maxima $10 \mathrm{~min}$ and $25 \mathrm{~min}$ after $3 \mathrm{rd}$ and 2 nd contact, respectively, which is a similar time window compared to the findings in this work.

\subsection{Environmental Studies}

Solar eclipses can have effects on terrestrial winds, temperature and weather in general. For example during the 11 August 1999 total solar eclipse transient a temperature decreases of up to $3{ }^{\circ} \mathrm{C}$ and a mean regional wind speed decrease of $0.7 \mathrm{~m} \mathrm{~s}^{-1}$ during the maximum eclipse hour with a mean anticlockwise wind direction change of $17^{\circ}$ were observed (Gray and Harrison 2012). Also the electric parameters of the atmosphere change: the ambient electric field dropped by up to $65 \%$ during the eclipse, and potential gradient showed epochs of enhancements during and after the eclipse until after Sunset (Anil Kumar et al. 2013). The authors conclude that during the course of an eclipse, radiative cooling enables the formation and development of water droplets resulting in scavenging or coalescence of free ions/free space charges in the atmosphere causing the decreases in electric currents and field strengths with the reduction in galactic cosmic rays flux as possible additional effect. Moreover it has been shown that the $\mathrm{pH}$ value of seawater during solar eclipse drops by $20 \%$ of the difference between ordinary water and seawater (Santhosh et al. 2014); and this drop is said to be due to the disputed effect of an eclipse on the ozone layer (see 1.1). Also effects on the marine microbiome have been reported (Backus et al. 1963). For example, solar eclipse induced effects on the marine environment have been shown to be driven mainly by the change of underwater irradiance (Economou et al. 2006). In another study it was shown that locking of the Sun ray during an eclipse at sea seems to promote the progeny of microbial predators in the race of better acclimatization and survival in the natural and changing environmental conditions (Shriyan et al. 2011).

\section{Theoretical Aspects}

\subsection{Surface Tension}

Surface tension is the elastic tendency of a fluid surface to create the least surface area possible. The field dates back to the days of Galileo (Galilei 1612); the first papers were written by Young (1805); a good overview can be found in the review by Good (1992). Briefly, surface tension is caused by cohesive forces among liquid molecules. In the bulk each molecule is pulled equally in every direction by neighboring liquid molecules, resulting in a net force of zero, whereas at the surface there is a net force pointing inwards. This force creates internal pressure and is responsible for liquid surfaces to contract to the smallest area possible. One method to measure this force is called the "Wilhelmy plate method" (Wilhelmy 1863; Adamson and Gast (1967). Here a thin plate is submerged into the liquid perpendicular to the interface and carefully pulled out until the retracting force reaches a maximum. This position is then kept constant, and the force exerted on it is measured with a microbalance using the Wilhelmy equation,

$$
\gamma=\frac{F}{l \cos \theta}
$$


where $\gamma$ is the surface tension, $F$ the force, $l$ the wetted perimeter and $\theta$ the contact angle between liquid phase and the plate. This method has been used successfully for several decades (Neumann et al. 1979; Jordan and Lane 1964; Lane and Jordan 1970; Princen 1970; Penn and Miller 1980; Bayramli et al. 1981; Seebergh and Berg 1992; Hayes and Ralston 1992, 1993a, b; Sauer and Kampert 1998). The apparatus used in this study, a Kibron AlphaPi Plus automatic tensiometer, uses a small needle instead of a plate and assumes complete wetting $(\theta=0)$, which simplifies the measurement so that the downwards force measured by the instrument's built-in micro balance becomes directly proportional to the surface tension.

\subsection{Astronomical Facts About Solar Eclipses}

A solar eclipse is a perceived darkening of the Sun that occurs when the Moon passes between the Sun and Earth whereby fully or partially blocking the Sun's light. Such a situation can only occur at new Moon, when the Sun and the Moon are in conjunction as seen from the Earth in an alignment astronomically referred to as syzygy. In a total eclipse, the disc of the Sun is fully covered by the disc of the Moon. In partial and annular eclipses, only part of the Sun is obscured. The event is therefore characterized by the optical coverage $C$, the ratio of the area of the solar disc occulted by the lunar disc compared to the total area of the solar disc, $C=A_{\text {Sun,obscured }} / A_{\text {Sun,total }}$. A complete derivation of this parameter for the physics relevant for the MSW effect is given elsewhere (Narayan et al. 1999). Other important parameters for an eclipse are the ratio of the apparent radii of the Moon and the Sun's discs seen from Earth, $a=r_{m} / r_{s}$ and the impact parameter $p$ defined as the apparent distance of closest approach between the center of the Sun's disc and the center of the Moon's disc. An eclipse is total if $a \geq 1$ and it is annular (as in the present case) if a $<1$. For an annular solar eclipse the Moon has to be in or close to its apogee. The progress of an eclipse can be divided into four stages:

(I) First contact: the Moon's disc starts to impinge on the Sun's disc $(C>0)$

(II) Second contact: the Moon's disc has moved completely within the Sun's disc ( $C=100$ for a total eclipse, and $C=94.33 \%, C=97.31 \%$ and $C=35.52$ for the annular eclipses in Réunion and Puerto Chacabuco and the partial eclipse in Rothera, respectively)

(III) Third contact: the Moon's disc starts to move out of the Sun's disc

(IV) Fourth contact: the Moon's disc leaves the Sun's disc entirely

These stages are marked in the following figures using the same Latin numbers as above. For the annular eclipse studied in this work the coverage $C$ can be calculated as follows (Narayan et al. 1999):

$$
C(d)=f(u)+a^{2}\left[1-f \frac{u}{a}\right]
$$

where

$$
f(u)=\frac{1}{\pi}\left(\arcsin u-u \sqrt{1-u^{2}}\right),
$$

and $d$ is the apparent separation between the centers of the Sun and the Moon (in units of solar radii), 


$$
d(t)=\left\{p\left[1-\left(\frac{2 t}{T}\right)^{2}\right]+\left(1+a^{2}\right)\left(\frac{2 t}{T}\right)^{2}\right\}^{\frac{1}{2}}
$$

with $t$ being the time (set to 0 for the point of maximum coverage) and $T$ the time between first and fourth contact.

For the calculation of the coverage in this work a computer program was used (WinEclipse and copyright 2002). The paths of the lunar shadow for the three eclipses are depicted in Fig. 1., relevant data about the eclipses is summarized in Table 1.

\section{Experimental}

\subsection{Réunion}

Experiments took place at the Observatoire Les Makes, 18, rue G. Bizet, Les Makes, 97421 La Rivière, Ile de La Réunion. An HP laptop running Windows 7 was used to control all devices and store the data. The water used in these experiments was "EDENA l'eau pure du cirque de mafate", a normal potable water available on the island. The ion content is given in Table 2. This water was chosen over distilled or purified water due to its stability concerning ion strength and $\mathrm{CO}_{2}$ content and associated parameters such as surface tension or refractive index, thereby minimizing a measurement drift induced by chemical reactions.

Temperature and humidity inside the lab were measured using a TSP01 2-channel compact USB temperature and humidity logger with an accuracy of $0.05{ }^{\circ} \mathrm{C}$ and $0.1 \%$ relative humidity, respectively (Thorlabs GmbH, Dachau, Germany). The measurement rate was one per second.

Refractive index measurements were conducted using a Rudolph J47-WR S5 refractometer in the continuous measure mode connected to the laptop via a virtual COM port. The J47 has a built-in temperature correction using the latest ICUMSA tables (ICUMSA
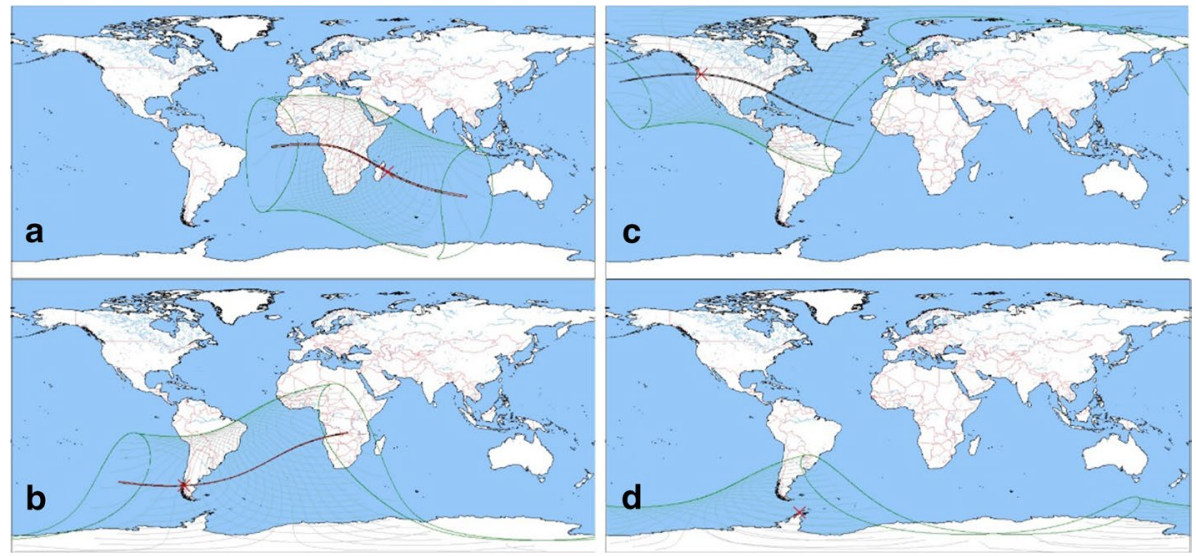

Fig. 1 Paths (red lines) of the lunar shadow for the solar eclipses in Réunion (a), Puerto Chacabuco (b), Oregon (c), and Rothera (d). Image created with WinEclipse software (Win-Eclipse and copyright 2002) 


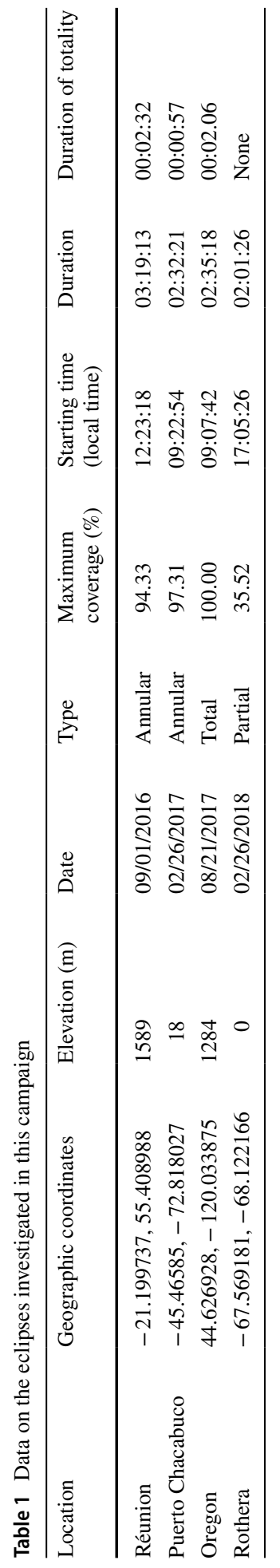


Table 2 Ion content of the sample water EDENA eau de source (2016)

\begin{tabular}{lclc}
\hline Cations & $c\left(\mathrm{mg} \mathrm{L}^{-1}\right)$ & Anions & $c\left(\mathrm{mg} \mathrm{L}^{-1}\right)$ \\
\hline $\mathrm{Ca}^{2+}$ & 11.2 & $\mathrm{HCO}_{3}^{-}$ & 79.3 \\
$\mathrm{Mg}^{2+}$ & 8.1 & $\mathrm{SO}_{4}{ }^{--}$ & 2.2 \\
$\mathrm{Na}^{+}$ & 8.2 & $\mathrm{Cl}^{-}$ & 6.5 \\
$\mathrm{~B}^{3+}$ & 6.0 & $\mathrm{NO}_{3}^{-}$ & 5.7 \\
& & $\mathrm{~F}^{-}$ & $<0.1$ \\
\hline
\end{tabular}

Methods Book and Bartens 2016). The accuracy of the measurements was $0.0001 \mathrm{nD}$, the measurement rate was one per $5 \mathrm{~s}$. Before the actual measurements the instrument was calibrated using Milli-Q water (one-point calibration). For the measurements, several $\mathrm{mL}$ of the sample water were put onto the J47 sapphire window, then the lid was closed to prevent interference from outside light sources and to minimize evaporation.

Background magnetic fields were measured using a 3-axis gaussmeter (Alphalab Vector/Magnitude Gauss Meter Model VGM). The model VGM is designed for rapid identification and measurement of areas that are magnetized. It is a DC gaussmeter with $\mathrm{X}, \mathrm{Y}$ and $\mathrm{Z}$ sensors at the end of a probe with a range from 0.00 to 799.99 gauss in 0.01 gauss steps, noise level (jitter) of 0.02 gauss for $\mathrm{X}, \mathrm{Y}$ and $\mathrm{Z}$ and magnitude (compare to the Earth field of about 0.5 gauss); and a $1 \%$ accuracy according to the manufacturer. All data (X, Y, Z and magnitude) were sampled at a $2 \mathrm{~Hz}$ rate.

Surface tension measurements were carried out using an AquaPi Plus tensiometer (Kibron Inc., Helsinki, Finland) with an accuracy of $0.1 \mathrm{mN} / \mathrm{m}$. Surface tension was measured using the system's Wilhelmy method (Wilhelmy 1863; Adamson and Gast 1967). In this method the needle is first submerged into the sample (by moving the sample) and then slowly pulled out until the point where pulling force does not increase any further. At this position the sample is held in place and the force is measured continuously at a rate of 10 values per second. Since the software delivered with the instrument was not designed for long-term measurements software was developed using said Wilhemy method which would store all measured data directly to the hard disk. Before the measurement the system was calibrated using two points (water and air) with 72.8 and $0.0 \mathrm{mN} / \mathrm{m}$, respectively. After initial stabilization of the system (variation of the readout $<5 \%$ ) average and standard deviation values of 200 single measurements were recorded. Whenever the standard deviation of that measurement was larger than $0.05 \mathrm{mN} / \mathrm{m}$ the measurement period was extended until such a precision was reached. Because the Kibron AquaPi Plus sample chamber is not gastight after some minutes of measurement the value started to drop due to evaporation of the sample. This process led to a detachment of the needle from the surface after 6-7 h. This time period was nevertheless long enough to measure continuously during the whole solar eclipse, from first contact to fourth.

The AquaPi + tensiometer shows very stable operation when measuring for the normal duration of surface tension measurements which is a few minutes at most. When measuring over hours, a linear drift can be observed, mostly downwards with different inclinations. The same drift is visible when just the weight of the needle is recorded, allowing the conclusion that the reason for this behavior is an electric drift of the balances of the tensiometer. For that reason, all surface tension measurements have been baseline corrected before testing their correlation to the eclipse. 
Table 3 Ion content of the sample water in Puerto Chacabuco (2017)

\begin{tabular}{llll}
\hline Cations & $c\left(\mathrm{mg} \mathrm{L}^{-1}\right)$ & Anions & $c\left(\mathrm{mg} \mathrm{L}^{-1}\right)$ \\
\hline $\mathrm{Ca}^{2+}$ & 2.3 & $\mathrm{HCO}_{3}^{-}$ & 47.3 \\
$\mathrm{Mg}^{2+}$ & 0.3 & $\mathrm{SO}_{4}^{2-}$ & 36.76 \\
$\mathrm{Na}^{+}$ & 49.0 & $\mathrm{Cl}^{-}$ & 38.66 \\
$\mathrm{~K}^{+}$ & 2 & $\mathrm{NO}_{3}^{-}$ & 1 \\
Other parameters & & & \\
$\mathrm{SiO}_{2}$ & 8 & $\mathrm{pH}$ & 8.5 \\
Hardness & 6.95 & $\mathrm{TDS}$ & 218 \\
\hline
\end{tabular}

\subsection{Puerto Chacabuco}

Experiments took place at a hotel room in the Loberias del Sur hotel, Jose Miguel Carrera 50 XI region, Puerto Chacabuco, Chile, since no scientific institute was available in the totality zone. An HP laptop running Windows 7 was used to control all devices and store the data. The water used in these experiments was "PUYEHUE Patagonia mineral water", a normal potable water available at the hotel. The ion content is given in Table 3. Temperature and humidity inside the hotel room were measured using the same equipment as in Réunion. Refractive index was not measured in Puerto Chacabuco. Surface tension measurements and DC magnetic field measurements were carried out as in Réunion. The air pressure was measured with a barometric pressure USB data logger type B1100-2, Gulf Coast Data Concepts, LLC, $1 \mathrm{~Hz}$ sample rate, $\pm 250 \mathrm{~Pa}$ absolute accuracy.

\subsection{Oregon}

Experiments took place in the open field in Oregon 97874, USA (exact coordinates latitude: 44.684277, longitude: - 120.033875), The open field was chosen for reasons of maximum duration and also to obtain one result without the possible interference of building roofs and walls. A Dell XPS 159550 laptop running Windows 10 was used to control all devices and store the data. The water used in these experiments was "Arrowhead Mountain Spring Water", a normal potable water available at most supermarkets. The ion content is given in Table 4. Temperature and humidity at the measurement site were recorded using the same equipment as in Réunion. Refractive index was again not

Table 4 Ion content of the sample water in Oregon (2017)

\begin{tabular}{llll}
\hline Cations & $c\left(\mathrm{mg} \mathrm{L}^{-1}\right)$ & Anions & $c\left(\mathrm{mg} \mathrm{L}^{-1}\right)$ \\
\hline $\mathrm{Ca}^{2+}$ & $4-66$ & $\mathrm{HCO}_{3}^{-}$ & No report \\
$\mathrm{Mg}^{2+}$ & $1.4-19$ & $\mathrm{SO}_{4}{ }^{2-}$ & $0-56$ \\
$\mathrm{Na}^{+}$ & $3-17$ & $\mathrm{Cl}^{-}$ & $0-12$ \\
$\mathrm{~K}^{+}$ & $0-2.9$ & $\mathrm{NO}^{-}$ & $0-2.2$ \\
& & $\mathrm{~F}^{-}$ & $0-1.2$ \\
Other parameters & & & \\
Hardness & $16-240$ & $\mathrm{pH}$ & $7.2-8.2$ \\
\hline
\end{tabular}


measured. Surface tension measurements were carried out as in Réunion. The air pressure and DC magnetic fields were not measured during this eclipse.

\subsection{Rothera}

Experiments took place at the Bonner labs at the Rothera research station in Antarctica. An HP laptop running Windows 7 was used to control all devices and store the data. The water used in these experiments was "cachantum ORIGEN NATURAL" mineral water, a normal Chilean potable water. The only ion content mentioned on the bottle is a sodium concentration of $20 \mathrm{mg} / \mathrm{L}$. Temperature and humidity inside the lab were measured using the same equipment as in Réunion. Refractive index was not measured at Rothera. Surface tension measurements were carried out as in Réunion, but this time also with a second tensiometer (Aqua Pi + , Kibron, newer model with micro USB interface) in parallel. The second tensiometer, although bearing the same name, had a higher sensitivity and an increased digital resolution, but was also more unstable and had higher relative errors. The air pressure was measured with a barometric pressure USB data logger type B1100-2, Gulf Coast Data Concepts, LLC, $1 \mathrm{~Hz}$ sample rate, $\pm 250 \mathrm{~Pa}$ absolute accuracy. In addition, the background magnetic field (AC) was measured using an AC Milligauss Meter Model UHS2 in a frequency range of $13 \mathrm{~Hz}$ to $75 \mathrm{kHz}$. The meter measures the true 3 -axis magnitude of the $\mathrm{AC}$ field. The background electric field was measured using a Trifield Natural EM Meter NEM which can detect small changes $(3 \mathrm{~V} / \mathrm{m})$ in the natural DC fields and some radio transients.

\subsection{Remark on Instrument Precision}

After the end of the measurement campaign the authors discovered an issue with the data transfer routines for the surface tension meters and the magnetometers which had resulted in the occasional addition of artificial noise to the measurement data, visible in the large error bars of some data points, but fortunately not so frequent or large that would obscure the effects reported. The authors could correct this issue to ensure that planned future measurements on this phenomenon will be more precise than the ones presented.

\section{Results}

In all locations a positive cross-correlation between the eclipse and the baseline-corrected surface tension was found. These results are summarized in Table 5.

Table 5 Cross-correlation of the baseline-corrected surface tension measurements with the eclipse at the respective locations during the solar eclipse experiments

\begin{tabular}{lll}
\hline Location & Correlation coefficient & $\begin{array}{l}\text { Time } \\
\text { shift } \\
(\mathrm{min})\end{array}$ \\
\hline Réunion & 0.656 & 20 \\
Puerto Chacabuco & 0.787 & 85 \\
Oregon & 0.815 & 30 \\
Rothera (1) & 0.796 & 40 \\
Rothera (2) & 0.690 & 35 \\
\hline
\end{tabular}


In all locations except for Oregon reference measurements were done at the same place at the same time but one day before or after the eclipse. As well as surface tension, environmental parameters (temperature, humidity and air pressure) were recorded. Depending on availability and equipment functionality not all parameters could be measured at all locations. Whenever there are no error bars visible the standard deviation is smaller than the diameter of the gray dot. The linear baseline drift was taken into account in all graphs by subtraction of said baseline. The baseline itself was determined from the initial behavior of the surface tension before the eclipse or, in case of the Rothera measurements, where the behavior itself was much more unstable than anywhere else, as a linear fit over the whole measurement. After starting the measurement the tensiometer needs a few minutes for stabilization (see e.g. steep rise Puerto Chacabuco data without eclipse, Fig. 5); these data points were not considered in the baseline calculation. The (spread of the) scales for all parameters are the same in the following graphs in order to facilitate comparison, except for Oregon where the temperature drop increased the surface tension rise almost by a factor of 2 (Figs. 2, 3, 4).

In Puerto Chacabuco also the evaporation rate of water, the weight of a filled (and sealed) and an empty water bottle were monitored during the eclipse. Whereas the weight of both the filled and the empty bottle did not change during the eclipse, the evaporation rate did, as is shown in Fig. 5.

The evaporation rate was calculated by differentiation of the weight data every $10 \mathrm{~min}$. It is noteworthy that the water evaporation rate peak, visible also as steepest increase in the water weight curve, coincides with the surface tension peak. This result is quantitatively reflected in the cross correlation analysis (see Table 6, Figs. 6, 7, 8, 9).

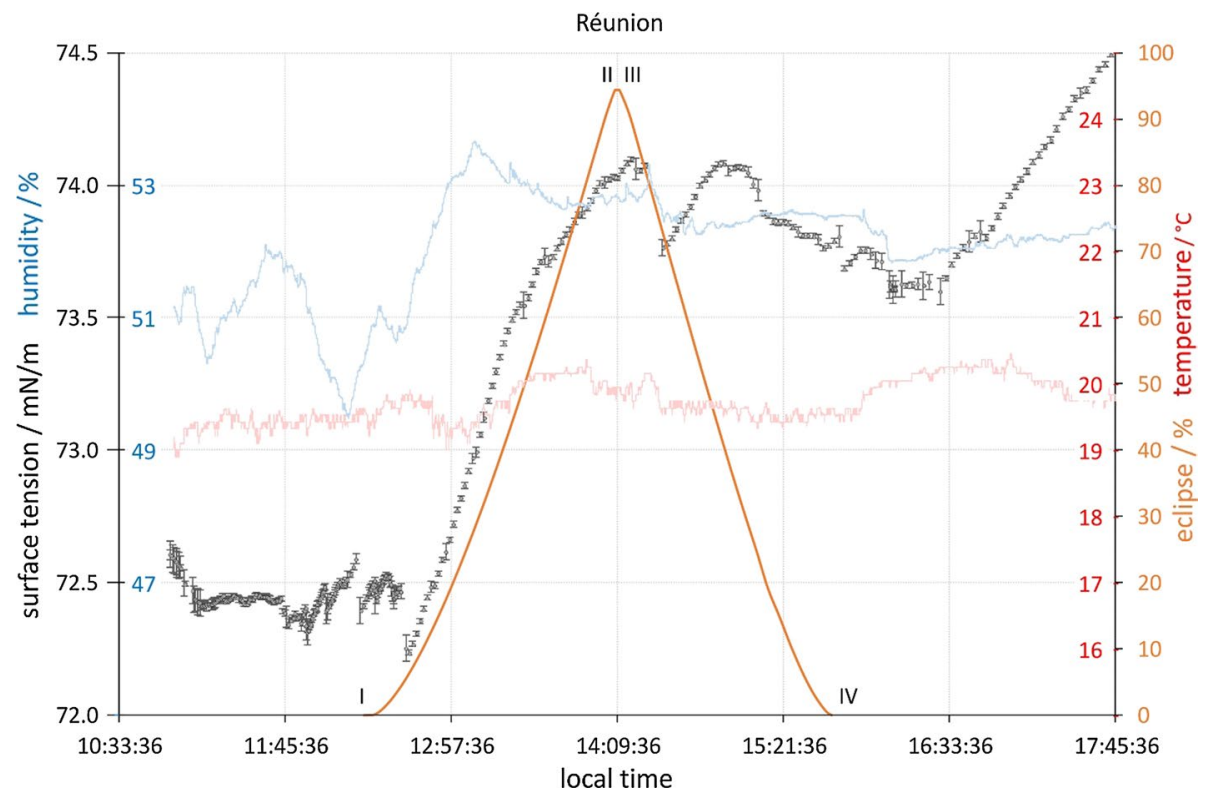

Fig. 2 Surface tension, temperature and humidity during the annular eclipse in Réunion. The scales for humidity and surface tension are on the left, the scales for eclipse and temperature are on the right side, respectively 


\section{Réunion}

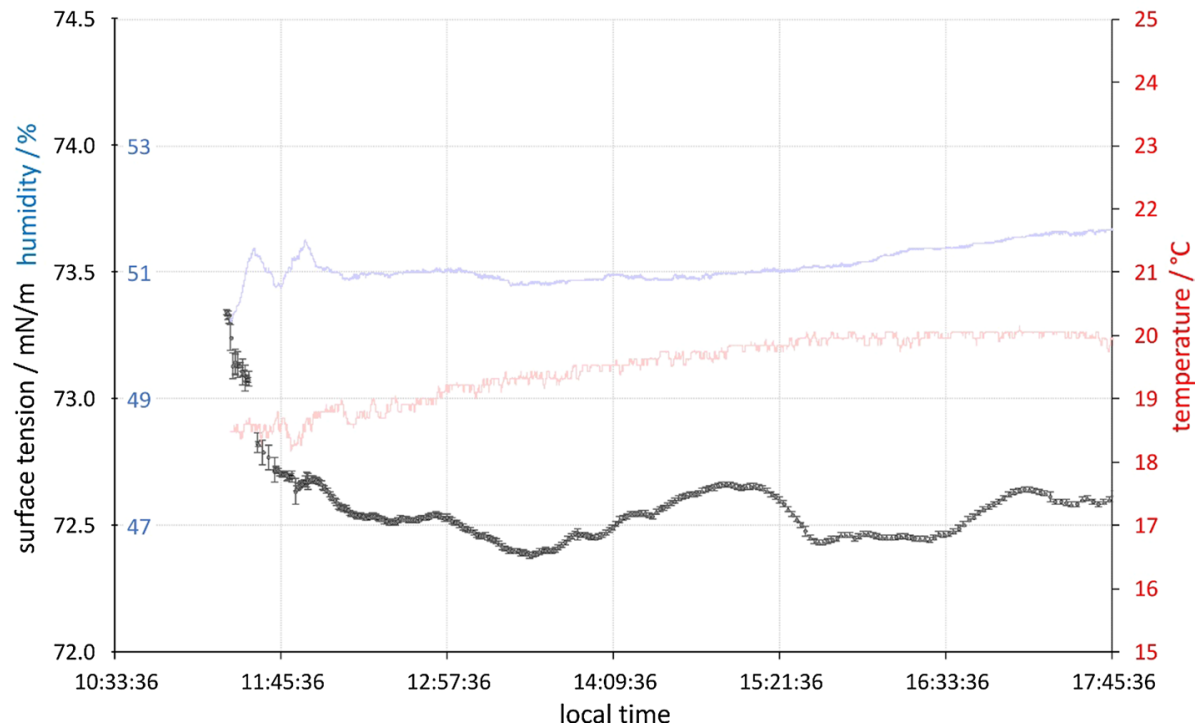

Fig. 3 Surface tension, temperature and humidity one day after the annular eclipse in Réunion. The scales for humidity and surface tension are on the left, the scale temperature is on the right side, respectively

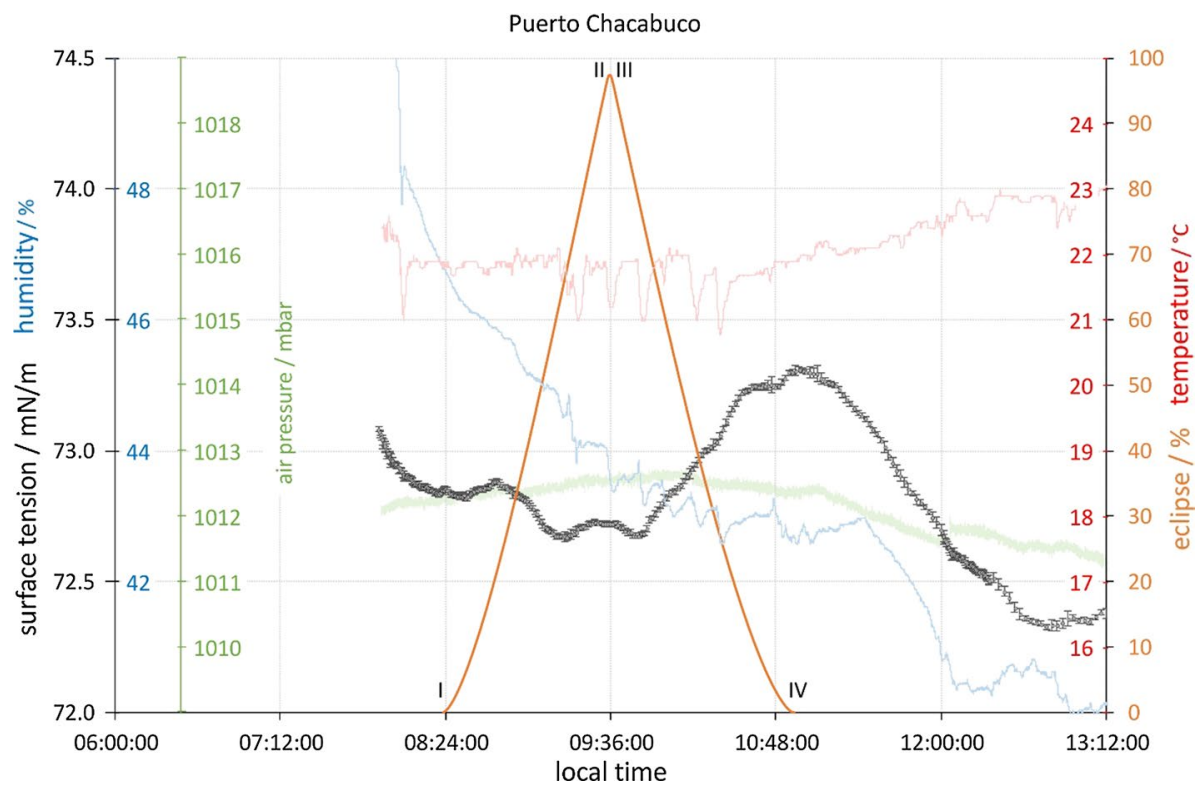

Fig. 4 Surface tension, temperature, humidity and air pressure during the annular eclipse in Puerto Chacabuco. The scales for humidity, surface tension and air pressure are on the left, the scales for eclipse and temperature are on the right side, respectively 


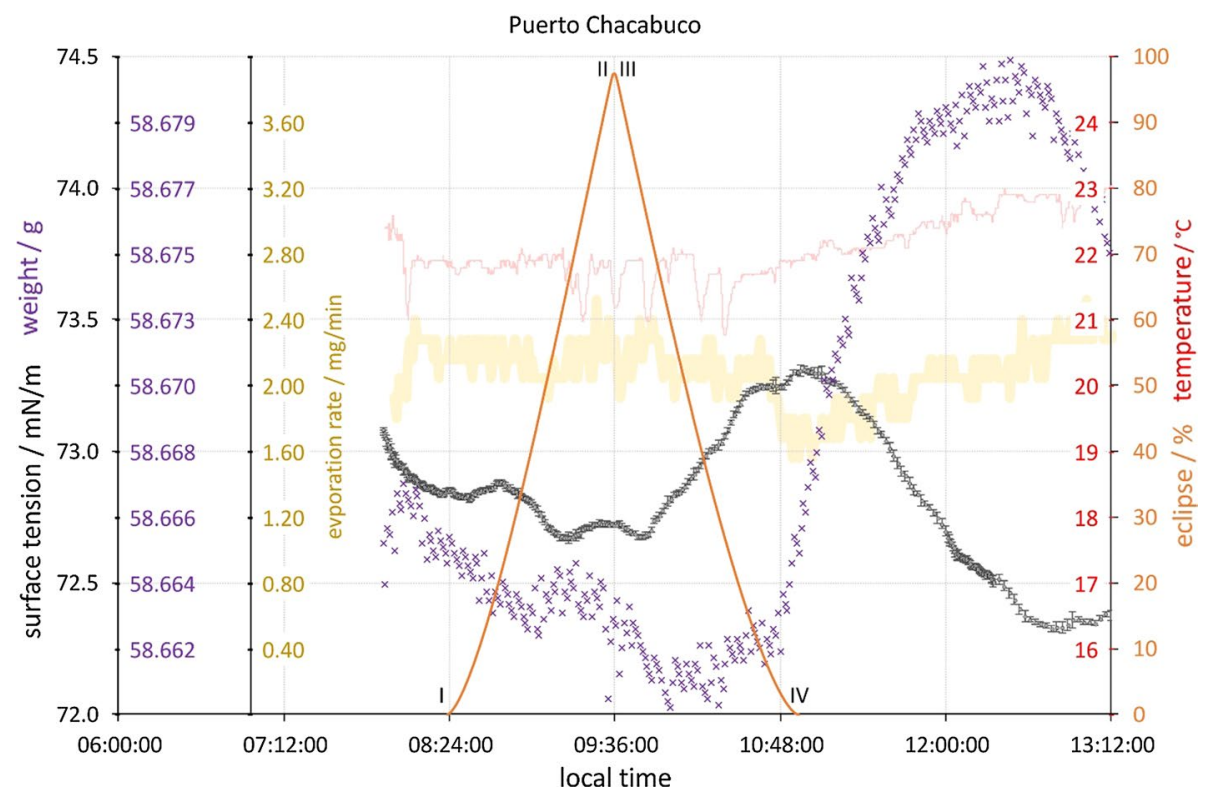

Fig. 5 Surface tension, temperature, weight of an open water bottle (purple crosses) and water evaporation rate (thick yellow line) during the annular eclipse in Puerto Chacabuco. The scales for surface tension, weight and evaporation rate are on the left, the scales for eclipse and temperature are on the right side, respectively

Table 6 Cross-correlation of the baseline-corrected surface tension and evaporation rate measurements with the eclipse in Puerto Chacabuco

\begin{tabular}{lcl}
\hline Measurement & Correlation coefficient & $\begin{array}{l}\text { Time } \\
\text { shift } \\
(\mathrm{min})\end{array}$ \\
\hline Surface tension & 0.787 & 85 \\
Evaporation rate & -0.724 & 88 \\
\hline
\end{tabular}

Refractive index, electric field and background radiation measurements did not show any correlation to the solar eclipse (data not shown). Additional correlations found in the data analysis are summarized in Table 7.

The results from all other measurements described in the experimental section did not reveal a correlation with the solar eclipses. 
Puerto Chacabuco

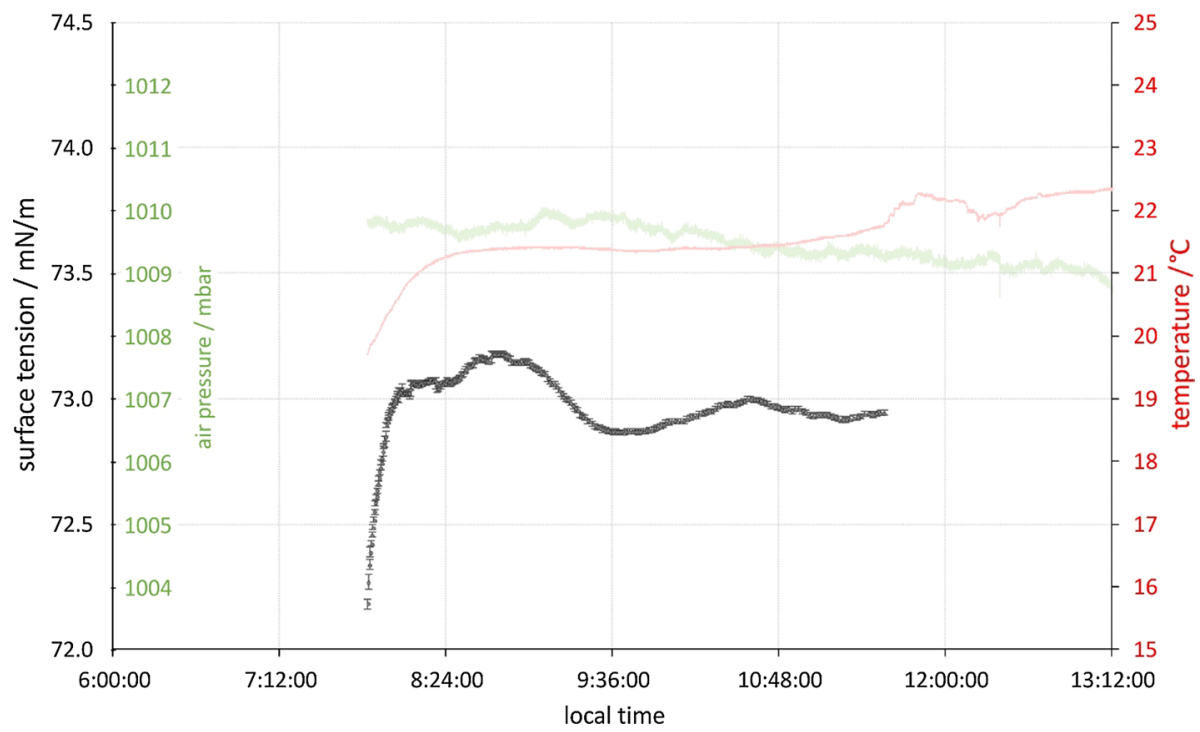

Fig. 6 Surface tension, temperature and humidity one day after the annular eclipse in Puerto Chacabuco. The scales for humidity and surface tension are on the left, the scale temperature is on the right side, respectively

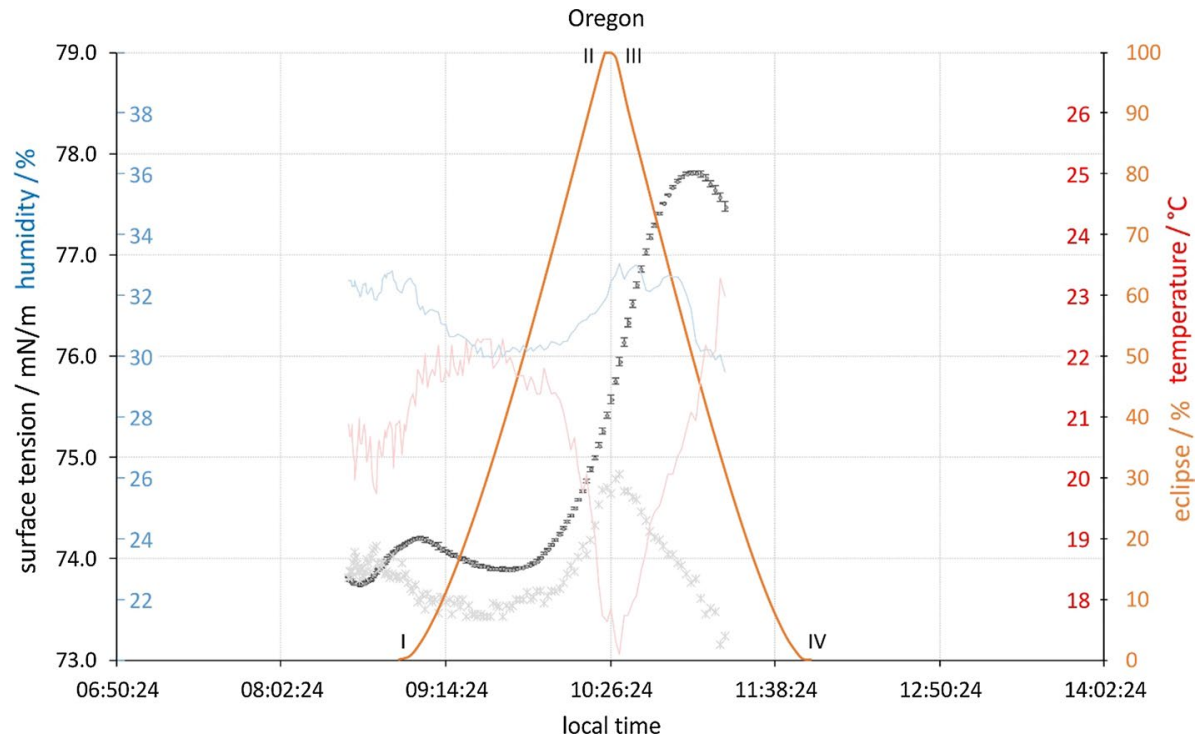

Fig. 7 Surface tension, temperature and humidity during the total eclipse in Oregon. The scales for humidity, surface tension and air pressure are on the left, the scales for eclipse and temperature are on the right side, respectively. The gray crosses show the calculated change of surface tension due to the temperature drop 


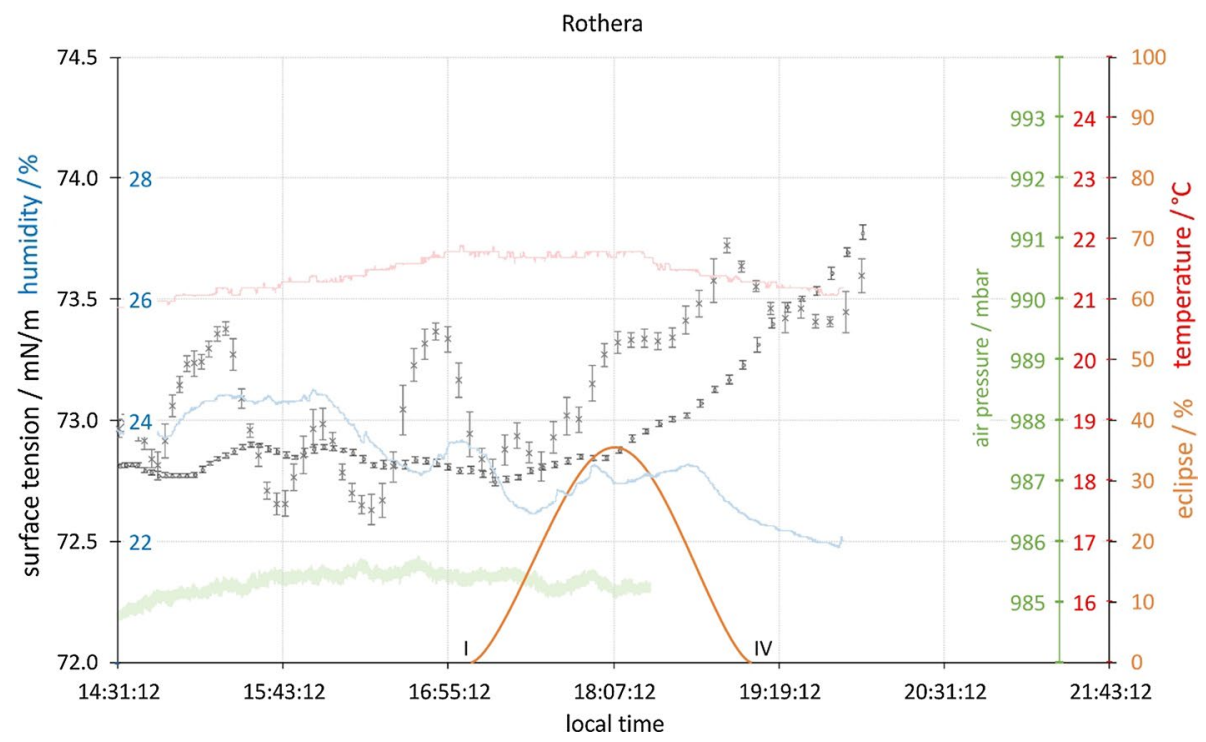

Fig. 8 Surface tension, temperature, humidity and air pressure during the partial eclipse in Rothera. The scales for humidity and surface tension are on the left, the scales for air pressure, eclipse and temperature are on the right side, respectively. The gray crosses are the data from the second, more sensitive tensiometer

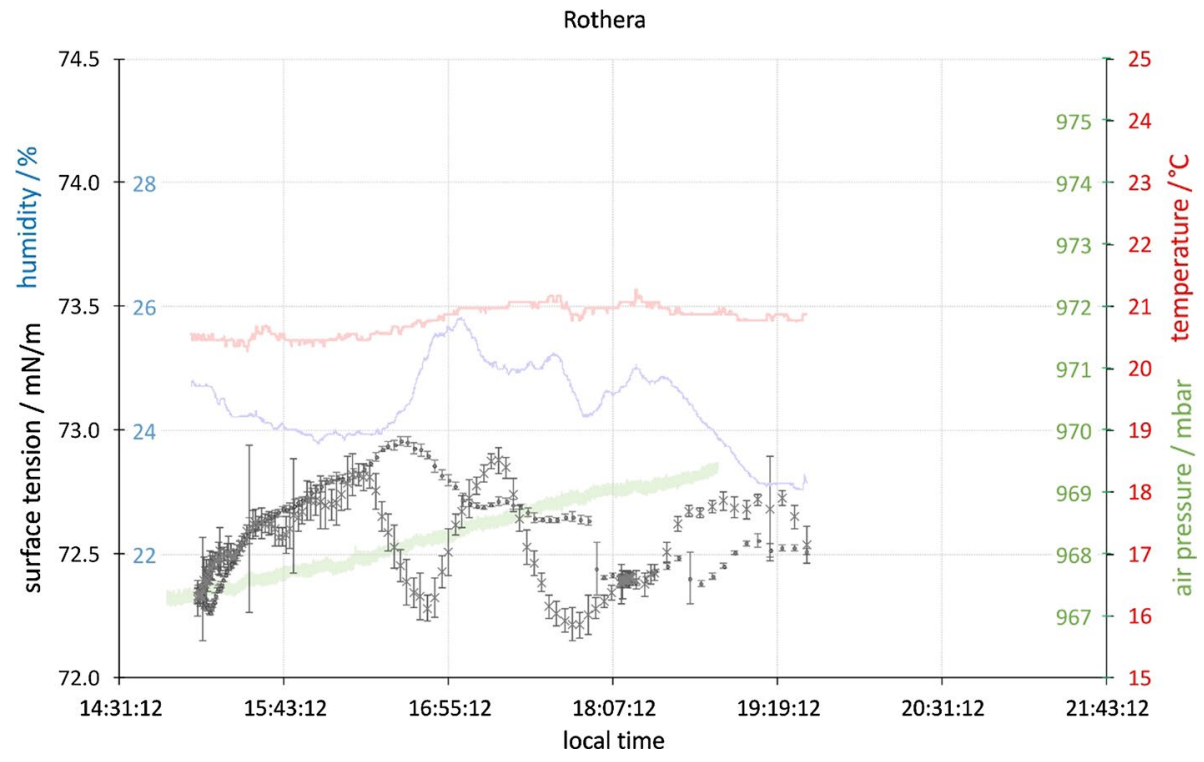

Fig. 9 Surface tension, temperature, humidity and air pressure one day before the partial eclipse in Rothera. The scales for humidity and surface tension are on the left, the scales for air pressure and temperature are on the right side, respectively. The gray crosses are the data from the second, more sensitive tensiometer 
Table 7 Additional correlations $(|P|>0.5)$ of measured parameters with the eclipses

\begin{tabular}{llc}
\hline Measurement & Location & $\begin{array}{l}\text { Correlation } \\
\text { coefficient }\end{array}$ \\
\hline Pressure & Puerto Chacabuco & 0.573 \\
Temperature & Rothera & 0.569 \\
Humidity & Rothera & -0.574 \\
Magnetic field z-com- & Rothera & -0.528 \\
ponent & & \\
\hline
\end{tabular}

\section{Discussion}

Measuring and understanding a hitherto unknown effect can be a very difficult and delicate task. The force that causes the surface tension to change in correlation to the solar eclipse might not be constant in the absence of the eclipse, so getting good reference data is a challenge. Not knowing its nature makes it impossible to shield it, and the best option is to keep all parameters constant except for the eclipse. That, however, is also only possible within certain margins. Nevertheless, the results of the measurements presented show a general common behavior: the rise of the surface tension in correlation with the eclipse event, albeit between 20 and 85 min later.

\subsection{Steps and Linear Retreat of the Surface Tension Measurement}

A closer look at the surface tension data reveals some downward steps mostly accompanied by short periods of instability (see e.g. Figure 1, shortly after the maximum of the eclipse). These instabilities caused the instrument to continue measuring until a standard deviation of 0.05 was reached, since during these moments the values were fluctuating so much that a reliable readout $(\sigma<0.05)$ was not possible. Such events are visible in the data as "blank periods" followed by large, sudden jumps. They can be explained by a step-wise film retreat from the needle, a known phenomenon for droplet evaporation (McHale et al. 2005) and is due to irregularities and associated energy barriers on wetted surface. In the present case some of these irregularities are probably created during the experiment by microscale calcium carbonate crystallization on the needle or by vibrations. Especially on Réunion, an island with an active volcano, it is likely that little tremors caused the irregular behavior. For the overall increase of surface tension observed in this work, however, we conclude that these steps are irrelevant and represent an independent phenomenon unrelated to the solar eclipse since they occurred both during and after the eclipse and also during both increase and decrease of the surface tension during the eclipse.

\subsection{Terrestrial Parameters Capable of Influencing the Surface Tension of Water}

\subsubsection{Humidity}

Pérez-Díaz et al. (2012) have shown that the effective surface tension on a water droplet decreases as humidity in the air increases. Their experimental data is in agreement with their model based on Newton's laws leading to 
Table 8 Correlation of the baseline-corrected surface tension measurements with humidity at the respective locations during the solar eclipse experiments

\begin{tabular}{lll}
\hline Location & $\begin{array}{l}\text { Correlation coef- } \\
\text { ficient }\end{array}$ & Time shift (min) \\
\hline Réunion & 0.672 & $20 \mathrm{~min}$ \\
Puerto Chacabuco & $<0.500$ & - \\
Oregon & $<0.500$ & - \\
Rothera (1) & $<0.500$ & - \\
Rothera (2) & $<0.500$ & - \\
\hline
\end{tabular}

$$
\gamma_{e}=\gamma+\frac{a(T)\left\langle V_{n}\right\rangle}{2 K}(1-h),
$$

where $\gamma_{e}$ is the effective surface tension, $\gamma$ is the surface tension, $a(T)$ is a function of the temperature $T$ and $h$ is the unitary humidity valued from 0 to 1 . In this model, it is assumed that evaporating molecules of water are ejected from liquid to gas with a mean normal component of the speed of "ejection" $\left\langle v_{n}>\right.$ greater than zero. As a result, depending on the temperature, the surface tension of water decreases with increasing humidity (negative correlation). At $20{ }^{\circ} \mathrm{C}$ the value ranges from $\sim 75.5 \mathrm{mN} / \mathrm{m}$ at $20 \%$ humidity linearly down to $73 \mathrm{mN} / \mathrm{m}$ at $100 \%$ humidity. Thus a humidity induced change in surface tension would be visible as a negative correlation. Table 8 shows the correlation of baseline-corrected surface tension and humidity for the four locations.

If humidity were (also) responsible for the observed effects, humidity drops of $20 \%$ negatively correlated to the surface tension changes should have been observed. That was not the case for any of the experiments performed. On the contrary, a moderate positive correlation was found for the measurements at Réunion, which probably weakened the observed effect.

\subsubsection{Temperature}

Next to humidity, from Fig. 1 of Pérez-Díaz et al. (2012) the temperature dependence of surface tension can be extracted using linear regression. In the present experiments, the humidity was different for each location, ranging between close to $50 \%$ in Réunion to about $20 \%$ in Rothera. The corresponding temperature coefficients for this humidity range are shown in Table 9.

In principle, as with humidity, there is a negative correlation between temperature and surface tension. The correlations found in the present experiments are given in Table 10 .

Table 9 Temperature coefficients according to (Pérez-Díaz et al. 2012) obtained by linear regression from measurements at $5,10,15$ and $20^{\circ} \mathrm{C}$

\begin{tabular}{lll}
\hline Humidity/ \% & Coefficient $(\mathrm{mN} / \mathrm{m}) /{ }^{\circ} \mathrm{C}$ & $\mathrm{R}^{2}$ \\
\hline 20 & -0.3018 & 0.999 \\
30 & -0.2818 & 0.999 \\
40 & -0.2504 & 0.999 \\
50 & -0.2298 & 0.988 \\
\hline
\end{tabular}


Table 10 Correlation of the baseline-corrected surface tension measurements with temperature at the respective locations during the solar eclipse experiments

\begin{tabular}{lcl}
\hline Location & Correlation coefficient & Time shift (min) \\
\hline Réunion & 0.541 & $20 \mathrm{~min}$ \\
Puerto Chacabuco & $<0.500$ & - \\
Oregon & $<0.500$ & - \\
Rothera (1) & $<0.500$ & - \\
Rothera (2) & $<0.500$ & - \\
\hline
\end{tabular}

If temperature was responsible for the observed effects, a negative correlation to the surface tension changes should have been observed. That was not the case for any of the experiments performed. On the contrary, again a moderate positive correlation was found for the measurements at Réunion, which probably weakened the observed effect.

In Oregon, where the experiments took place in the field, both temperature and humidity in that experiment changed drastically during the eclipse. There is also correlation of the eclipse with temperature $(P=-0.623)$. In order to estimate how much of the effect is due to the temperature change, the theoretical change of the surface tension due to the temperature drop at the measured humidity was calculated and shown as grey $x$ symbols. The temperature coefficient for each data point was obtained from a linear regression from the data in Table 9 and the humidity data. About one-third of the surface tension rise can indeed be explained by the temperature drop.

Since humidity and surface tension are negatively correlated and the humidity rose during the experiments in Oregon, any correction for a humidity change would only slightly increase the observed surface tension rise, but cannot be the cause of it.

\subsubsection{Air Pressure}

Van Flandern and Yang (2003) have shown that rapid air mass movement for the bulk of the atmosphere above normal cloud levels increased the atmospheric pressure by at least $0.6 \%$ during an eclipse (from 979 to $985 \mathrm{~g} / \mathrm{cm}^{2}$ ) and returned to pre-eclipse level afterwards. This change was induced by rapid air mass movement for the bulk of the atmosphere above normal cloud levels and was said to be sufficient to explain gravitational anomalies reported previously (Van Flandern and Yang 2003). This change is much bigger than any air pressure change observed in the experiments in this work. Massoudi and King (Massoudi and King 1974) have investigated the effect of air pressure on the surface tension of water. According to their measurements, the interfacial tension as function of pressure for water can be approximated by a 3rd grade polynomial. Assuming the same relation can be used as good approximation for the potable water used in this study and an atmospheric composition of $78.08 \% \mathrm{~N}_{2}, 21.88 \% \mathrm{O}_{2}$ and $0.04 \% \mathrm{CO}_{2}$, a pressure drop of 9.8 atm would be necessary to explain the increase of surface tension observed in this work. Although air pressure was not monitored during all eclipses it is safe to say that such a drop did not occur, and that any atmospheric pressure increase as reported by Van Flandern and Yang (2003) would have caused a reduction of the surface tension in the $\mu \mathrm{N} / \mathrm{m}$ range, which is beyond the precision of the instrument used. A more direct approach is the calculation of the volume change due to air pressure change. With a compressibility of $\sim 5 \times 10^{-10} \mathrm{~Pa}$ it is straightforward to calculate that the volume of water would decrease by $0.25 \mathrm{ppm}$ due to the 5 mbar pressure increase. The sample beaker can be approximated by a cylinder of $1 \mathrm{~cm}$ height, which would be reduced by 
$2.5 \mathrm{~nm}$ due to the increase pressure. This distance is equivalent to a layer of about 10 water molecules and far beyond the precision of the instrument used in this study.

\subsection{Cosmic Parameters Capable of Influencing the Surface Tension of Water}

\subsubsection{Gravity and Tides}

The tidal acceleration at the Earth's surface along the Moon-Earth axis is about $1.1 \times 10^{-7} \mathrm{G}$ (Ministry of Defence (NAVY) 1987). According to a NASA report (Weislogel et al. 1998) the surface tension of water is dependent on gravity, and a linear interpolation of the values given in this report allows an estimation of the effect during a solar eclipse, which is an increase of $\sim 1.1 \times 10^{-7} \mathrm{mN} / \mathrm{m}$. This change is 6 orders of magnitude lower than the effect measured in this work.

Another possible cause would be the change of capillary rise due to said change of gravity. The height of a liquid column is given by Batchelor et al. (1967)

$$
h=\frac{2 \gamma \cos \theta}{\rho G r}
$$

where $\gamma$ is the surface tension, $\theta$ is the contact angle, $\rho$ is the density of liquid, $\mathrm{G}$ is the local acceleration due to gravity, and $r$ is the radius of tube. A change of gravity by $1.1 \times 10^{-7} \mathrm{G}$ results in a change of $h$ by $\sim 10^{-13} \mathrm{~m}$ independent from the other parameters. Or, a small change of, for example, $0.8 \mathrm{mN} / \mathrm{m}$ would require an increase of gravity by $0.11 \mathrm{G}$. The first effect is negligible, and the second is unrealistic and carries the wrong sign.

Finally, it may be mentioned that the water level in a small beaker cannot be directly compared to an ocean concerning tidal range. The huge tidal range in the oceans is possible because large bodies of water move from one location (where there is less attraction from the Moon) onto another (where there is lunar attraction)-water is not expanding or shrinking, its compressibility is extremely low $\left(\sim 5 \times 10^{-10} \mathrm{~Pa}^{-1}\right)$. In a beaker the circumstances are different. Displacement of water is not possible, and the volume expansion is negligible, and tilting of the surface would also be in the nm range. In addition this change will take place over a period of $12 \mathrm{~h}$ in a sinusoidal manner, so the change during the period of an eclipse, when it will be at the max of the sinusoidal variation will be even more negligible.

\subsubsection{Solar Wind}

The continuous flow of particles emanating from the Sun and permeating the solar system is often referred to as solar wind. It consists mostly of electrons, protons and helium nuclei (alpha particles). Its existence was first suggested by Arthur Eddington (2006) and Biermann (1951) suggested it to be reason for a comet's tail pointing always away from the Sun independent from its heading. In 1959 solar wind was measured directly for the first time by the Soviet satellite Luna 1 (2007). The solar wind consists of about $1.3 \times 10^{36}$ particles per second (Kallenrode 2004) resulting in a total annual solar mass loss of $\sim(2-3) \times 10^{-14}$ solar masses (Carroll et al. 1995) equal to about a billion kilograms per second. The velocity distribution of the solar wind shows two maxima and is therefore divided into the slow solar wind and the fast solar wind, with their differences extending beyond their speeds. In 
near-Earth space the slow solar wind has a velocity of $300-500 \mathrm{~km} \mathrm{~s}^{-1}$ and a composition comparable to the corona. The fast solar wind has a typical velocity of $750 \mathrm{~km} \mathrm{~s}^{-1}$ and its composition is comparable to the Sun's photosphere (Geiss et al. 1995). The slow solar wind is twice as dense and more variable in nature than the fast solar wind (Kallenrode 2004; Suess 1999).

The temporal offset of the surface tension effect suggests a dependence on a cause that travels from Moon to Earth significantly slower than the speed of light, and on the first glance solar wind seems to be a promising candidate. The observed time differences yield velocities between 193 and $386 \mathrm{~km} \mathrm{~s}^{-1}$, which is at the lower end of the slow solar wind component. However, predicting the occurrence of a solar wind shadow precisely is not a simple task: When a syzygy of Earth, Moon and Sun results in an eclipse the solar wind is passing by the Moon, which is creating a shadow. However, the slow solar wind component will take about 16 min to reach Earth. Whereas the solar wind shadow that was being cast at the time of eclipse moves towards the Earth, the Earth is sliding sideways as it orbits the Sun. After $16 \mathrm{~min}$, when the shadow reaches the position that the Earth was in at the time of the eclipse, the Earth has been travelling for $16 \mathrm{~min}$ on its orbit around the Sun at $\sim 30 \mathrm{~km} \mathrm{~s}^{-1}$ so will have moved about $28830 \mathrm{~km}$ or 2.3 Earth diameters. In a first approximation, as a result the Moon shadow would completely miss the Earth. However, solar wind consists of charged particles which are strongly deflected by the Earth's and Sun's magnetic fields and attracted (and repelled, depending on the charge) by the Earth's electric field. Without knowing the exact magnitude and geometry of these fields at the time of the eclipse it is impossible to predict whether a solar wind shadow will reach Earth or not.

The impact of the solar wind on Earth is measured by NASA satellites and commonly known as the so-called $K$-index. It quantifies disturbances in the horizontal component of Earth's magnetic field with an integer in the range 0-9 with 1 being a calm and 5 or more indicating a magnetic storm, and is derived from the maximum fluctuations of horizontal components observed on a magnetometer during a three-hour interval. The index was introduced already in Bartels et al. (1939). Table 11 summarizes the K-indices for the time periods of the solar eclipses studied in this work.

There is a seeming correlation between the K-index and the magnitude of the effect, corroborating the solar wind hypothesis, taking into account that the eclipse at Rothera was only partial. In addition, a solar eclipse in a region with high solar wind density such as Rothera should produce a stronger effect and a more strongly fluctuating base line. Again, taking into account that the eclipse in Rothera was only partial, both of these effects were found. Keeping in mind that a correlation alone can never prove a causality relation, the data at hand makes the solar wind hypothesis the most likely one of all hypotheses mentioned here. This statement, however, requires a model as how the solar wind physically changes the surface tension, which is presented in the next paragraph.

Table 11 K-indices and surface tension rise during solar eclipse events 2015-2018. The surface tension rise in Oregon due to the temperature decrease is subtracted from the total amount

\begin{tabular}{lll}
\hline Location & K-index & $\begin{array}{l}\text { Surface tension } \\
\text { rise }(\mathrm{mN} / \mathrm{m})\end{array}$ \\
\hline Réunion & 4.5 & 1.6 \\
Puerto Chacabuco & 0.5 & 0.6 \\
Oregon & 3.0 & 2.4 \\
Rothera & 3.0 & 1.0 \\
\hline
\end{tabular}


5.3.2.1 Terrestrial Muons Disrupt the H-bond Network At the Water/Air Interface Muons are abundantly produced by the interaction of high energy cosmic rays with Earth's upper atmosphere according to

$$
C R+A \rightarrow \pi+K \rightarrow \mu+v
$$

where CR are the cosmic rays, $A$ is Earth's atmosphere, $\pi$ are pions, $K$ kaons, $\mu$ muons and $v$ neutrinos. Kaons decay again into muons and neutrinos (Marinho et al. 2014). The cosmic ray flux has a broad energy spectrum extending from $10^{3}$ up to $10^{20} \mathrm{eV}$ (Yao et al. 2006), most of which, however, is perfectly shielded by the geomagnetic field (Abraham et al. 2010). Muons, however, are unaffected by this shielding since they are created inside Earth's atmosphere. Therefore they are the dominant component of the radiation flux at sea level for energies above $100 \mathrm{MeV}$ (Alpen 1998). Interestingly, it has been shown that the muon flux on Earth's surface is dependent on the K-index (Brancus et al. 2009), pointing at a correlation between the intensities of the solar wind and the cosmic radiation. Without going into detail about how exactly these two parameters are related, this finding (Brancus et al. 2009) allows to conclude a correlation between the muon density on Earth and the solar wind intensity measured by the K-index. Furthermore, it has been shown that when other cosmic rays shower in the Earth's atmosphere and create muons, there is a relative deficit of muons from the direction of the Moon (Boersma et al. 2009). In this hypothesis we suggest a similar "Moon shadow" event in conjunction with the solar wind and its relation to cosmic rays (Brancus et al. 2009).

The main reaction caused by muon interactions is ionization, giving rise to several electrons and low-energy photons that will propagate and interact further (Marinho et al. 2014). The energy necessary for ionizing a water molecule, along their paths through water is about $13 \mathrm{eV}$, thus many orders of magnitudes lower than the typical energies deposited by a muon, which are in the keV range. Muons leave a trace of secondary electrons, ionized water molecules, radicals and the products thereof along their penetration path, thereby creating additional charge carriers in the liquid. It has recently been shown that water with positive excess charge does exist (Fuchs et al. 2016) and that this charge will accumulate at the surface, lowering the surface tension (Fuchs et al. 2019). Moreover, it has been shown (Chen et al. 2016) that the ionisation of a single water molecule can result in collective effects which can be linked to macroscopically detectable distortions like the surface tension. These findings support the hypothesis that the absence of muons is the source for the surface tension rise shortly after a solar eclipse. If the solar wind and the cosmic radiation are blocked by the Moon during a solar eclipse event, the muon production in the upper atmosphere should be reduced as well. Fewer muons then impact on the water/air interface, which leads to fewer disruptions and a higher surface tension-and a lower evaporation rate as observed during the Puerto Chacabuco eclipse.

Considering that the average muon flux is about 10,000 muons $/ \mathrm{m}^{2}$ per minute (Grieder 2001), it is straightforward to calculate the number of molecules in the beaker, approximated as a perfect cylinder with $1 \mathrm{~cm}$ radius and height, with which these muons interact assuming total muon penetration and a density of $1 \mathrm{~kg} / \mathrm{dm}^{3}$ ), which is $1.1 \times 10^{8}$ molecules/ minute. We could now assume that time lag of the surface tension rise is partly due to velocity of the solar wind and partly due to the dynamics of the muons in solution. The average solar wind velocity close to Earth is roughly $500 \mathrm{~km} / \mathrm{s}$, which could explain about 13 min of the delay. If we average the time lags of the sites with similar K-index (all except Puerto Chacabuco), the average lag time is $(31 \pm 4) \mathrm{min}$ (standard error), which would mean that it takes $18 \mathrm{~min}$ for muon influence to wear of, or to start again. This time constant is comparable to the time constant for discharging protonically charged water (22 min) (Fuchs 
et al. 2019). That effect would increase the number of ionized molecules causing the effect to $2 \times 10^{9}$, which is equivalent to a concentration in the order of 1 pico mol/L. Surface tension changes due to free charges have been found in the nano mol range (Fuchs et al. 2016). Thus, in order to explain the surface tension rise with this effect, we have to assume that the impact of a muon on a water molecule will influence (ionize) at least a thousand water molecules in the neighbourhood, and that the charged species created by this process will not immediately recombine, but survive in the liquid for a matter of minutes. The latter has been shown recently (Fuchs et al. 2016, 2019); and given the energy of a muon is in the $\mathrm{GeV}$ range, a mass ionisation event seems possible. Theories that support the idea of the coherent behaviour of thousands of water molecules that would allow such processes do exist (Del Giudice et al. 1985), and the authors are planning to devise a fully developed quantum field theory explanation describing in detail the interaction between a muon and a coherence domain. In addition, the authors plan to further test this hypothesis by measuring the muon intensity directly during an eclipse event.

For the sake of completeness, additional less likely (but nevertheless possible) hypotheses explaining the result are presented in the appendix.

\subsection{Summary}

We have shown that the blocking of the solar wind and cosmic radiation with the subsequent absence of atmospheric muons during an eclipse event is a plausible working hypothesis for the observed changes in surface tension and evaporation rate during solar eclipses. Alternative hypotheses including a a novel neutrino/water interaction are presented in the appendix. None of the obvious environmental parameters (temperature, humidity and pressure) can explain the effects observed.

It is noteworthy that many solar eclipse effects reported in literature, such as the hypothesized change in the ozone layer (Bezverkhny et al. 1956; Fournier 1956; Kawabata 1936; Khrigian et al. 1961; Jerlov et al. 1954; Pariisky and Chen-Chao 1958; Steblova 1961; Stranz 1961; Svensson 1968; Chakrabarty et al. 1997; Zerefos et al. 1999), or the pH of the sea (Santhosh 2014), happened at approximately the same time offset as the effects in our study, some tens of minutes after the eclipse. An influence much slower than the speed of light seems plausible at first glance, pointing at solar wind and cosmic radiation blocking as possible cause for these effects as well. Although the involved magnetic and electric fields make an actual simulation of the effect very difficult, it remains to be the most likely hypothesis.

\section{Conclusions}

Studies of surface tension of water during four solar eclipse events have resulted in an increase between 0.6 and $2.4 \mathrm{mN} / \mathrm{m}$ between 20 and $85 \mathrm{~min}$ after the maximum eclipse, respectively, independent of environmental parameters like temperature, air pressure and humidity. The magnitude and times can be correlated to the K-index which reflects the impact of solar activity on Earth. During one of the eclipses water evaporation experiments were conducted and showed a temperature independent reduction of water evaporation at the same time as the surface tension increase was observed. A disruption of the water/air interface by muons which is reduced during a solar eclipse due to blocking of both cosmic 
radiation and solar wind is presented as working hypothesis. As alternative hypothesis, we propose a novel neutrino/water interaction based on chiral preferences of water interactions. More studies and theoretical approaches are called for to further investigate and explain the phenomenon observed.

Acknowledgements Open access funding provided by Graz University of Technology. This work was performed at the Wetsus, European Centre of Excellence for Sustainable Water Technology (www.wetsu s.eu) as well Observatoire Les Makes, 18, rue G. Bizet, Les Makes, 97,421 La Rivière, Ile de La Réunion; the hotel Loberias del Sur, Jose Miguel Carrera 50 XI region, Puerto Chacabuco, Chile; Naresuan University, Thailand; Oregon 97874, USA, in the field (the exact coordinates were latitude: 44.684277, longitude: -120.033875 ) and Rothera base, Antarctica. Wetsus is co-funded by the Dutch Ministry of Economic Affairs and Ministry of Infrastructure and Environment, the Province of Fryslân, and the Northern Netherlands Provinces. Furthermore the authors hereby would like to thank the following persons for friendly cooperation and fruitful discussions about the subject: Maarten van de Griend, everybody at the Observatory in Les Makes; the hotel crew at hotel Loberias del Sur, the Chilean Military; the Netherlands Polar Programme and Dick van der Kroef; the British Antarctic Survey, Alison J. Massey, Nigel Bird and his team, Michael Prior-Jones, and the whole crew at Rothera; Jannie Mulder, John Swain, Giuseppe Vitiello, Astrid H. Paulitsch-Fuchs, Erich M. Wappis, Klaus Volkamer and Adam D. Wexler.

Open Access This article is distributed under the terms of the Creative Commons Attribution 4.0 International License (http://creativecommons.org/licenses/by/4.0/), which permits unrestricted use, distribution, and reproduction in any medium, provided you give appropriate credit to the original author(s) and the source, provide a link to the Creative Commons license, and indicate if changes were made.

\section{Appendix: Alternative Hypotheses and Additional Measurements}

\section{Solar Neutrinos}

\section{Solar Neutrino Deflection}

Next to electromagnetic radiation the Sun is a copious source of neutrinos with a wide spectrum of energies. A flux of $6 \times 10^{10}$ neutrinos $\mathrm{cm}^{-2} \mathrm{~s}^{-1}$ is present on the surface of the Earth as a result of nuclear reactions within the Sun (Bahcall and Pinsonneault 2004). High energy neutrinos from the Sun have been detected by terrestrial neutrino detectors like Super-Kamiokande, SNO, and Borexino (Totsuka 1996; Suzuki 1994; SNO Collaboration 1987; Raghavan 1990). The interaction cross section of such neutrinos is sufficiently small that the neutrino flux is not significantly reduced by its passage through the Earth, and the variation that is seen can be explained by neutrino oscillations (Smy et al. 2004). At first sight therefore it would not be expected that the Moon would cast a "neutrino shadow" on the Earth during a solar eclipse. However it has been proposed that scattering interactions of neutrinos by very hard crystalline material can be of the order $10^{20}$ greater than relatively soft solids as a result of coherent interactions (Lewis 1980; Barranco et al. 2005; Weber 1985). Such scattering has been demonstrated, which has included showing the scattering of the solar neutrino flux by a sapphire crystal (Nicolescu 2013; Weber 1988). Seismology data suggests the presence of a layer $65 \mathrm{~km}$ below the lunar surface that contains material such as the corundum (Anderson 1975). Such material would have exactly the characteristics required to diffract neutrinos such that the Moon would act as a neutrino diffuser, deflecting solar neutrinos from their original trajectory as they passed through the Moon. At a sufficiently large distance, such as the distance between the Earth and the Moon, the Moon would consequently cast a neutrino shadow, such that there would be a dramatic drop in the flux of solar neutrinos during a solar eclipse. 
While this would suggest a reasonable possibility that a solar eclipse would also be associated with a dramatic reduction in the solar neutrino flux, the neutrino interaction with water would not at first sight indicate the existence of a measurable interaction with water such that it could cause a change in surface tension. However, measurements indicate that there is a difference in the interaction between water and opto-isomers of molecules, for example as seen in the very small difference in the heat of solvation of $-\mathrm{D}-$ and $-\mathrm{L}$ optoisomers of molecules (Scolnik et al. 2006; Deamer et al. 2007). Theoretical physics provides only one possible explanation for such chiral differences: the process must at some level be associated with neutrinos in that they only exist as left chiral particles whereas all other fundamental particles exist as a symmetrical mixture of left and right chiral particles (Mason 1986). This is the explanation for the chiral dependence of beta nuclear decay as these involve the weak force and the emission of a left handed neutrino. While the mechanism behind the chiral preferences of water interactions are still poorly understood, it must be the case that these interactions involve neutrinos. This raises the possibility that a number of the properties of water, including the strength of surface tension forces, could be partly dependent on the solar neutrino flux. As such it would be expected that there would be slight variations in some of these properties during a solar eclipse.

\section{Solar Neutrino Flavor Change}

Mixing and the consequent oscillations among neutrinos of different flavors is generally believed to be the cause of the reduced intensity of neutrino flux detected on Earth. Neutrino mixing and oscillations were first discussed by Bruno Pontecorvo in a series of papers starting in Pontecorvo (1957, 1958, 1968). In Gribov and Pontecorvo (1969) suggested that neutrino oscillations due to lepton nonconservation might lead to a decrease of detectable solar neutrinos on earth. The possibility that neutrinos can change their quantum mechanical flavor in their interaction with matter was first discussed by Lincoln Wolfenstein (1978). In Mikheyev and Smirnov (1985) predicted that non-absorptive interaction with matter could resonantly enhance the neutrino mixing which was later experimentally proven (Parke 1986; Bethe 1986; Rosen and Gelb 1986). This effect is nowadays known as Mikheyev-Smirnov-Wolfenstein (MSW) effect and describes the change of quantum mechanical flavor when neutrinos pass through matter. Such an effect takes place during a solar eclipse when solar neutrinos pass through the Moon on their way to Earth (see Fig. 10). More precisely, this effect increases the number of measurable neutrinos (electron neutrinos) by a factor of 1.5 (Narayan et al. 1999). Because of the very low sensitivity of today's neutrino detectors it is unlikely that such an effect can be measured by conventional methods. Nevertheless, the authors who calculated this change stress "... the novelty of the whole phenomenon, and urge the experimentalists to look for and study the eclipse effects in an unbiased manner. They may even discover some surprises, not predicted by our calculations. ..." (Narayan et al. 1999). An interaction between the electron neutrinos and the water/air interface resulting in a measurable change in surface tension as observed in this work would certainly count as such a surprise. However, since neutrinos travel almost with the speed of light, the delay found in the present experiments would not be observable. 


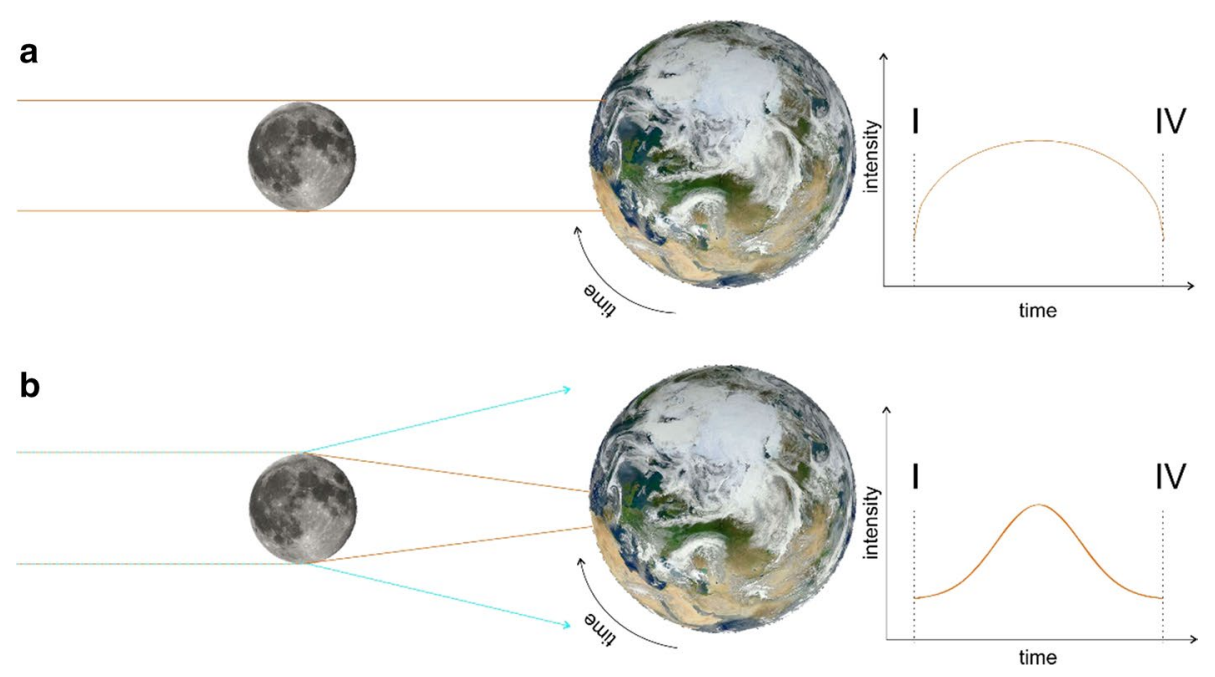

Fig. 10 Sketches of the path of rays for neutrinos (a) and dark matter focusing/dispersing (b) with their respective intensity profiles at the eclipsed region over time (right end). I and IV are first and fourth contact of the eclipse, respectively

\section{Double Eclipse Surface Tension Measurement}

The two alternative neutrino hypotheses both suggest that it might be possible to see a surface tension change at a "double eclipse" location (see Narayan et al. 1999) associated with a total eclipse. One of the authors did conduct a measurement in Naresuan University, Thailand at a "double eclipse" location on September 21st, 2017. No correlation of the surface tension curve with the double eclipse event was found (data not shown). These results indicate that neutrino flavor changes can probably be dismissed as a reason for the effects observed. The implication for the neutrino deflection hypothesis is less clear in that small deflections as the neutrinos enter the Earth through strata containing relatively hard minerals could cause diffusion that is sufficient to make the neutrino eclipse much less pronounced on the far side of the Earth.

\section{Solar Dark Matter Quanta}

An even more speculative hypothesis concerns the interaction of water with a recently proposed type of dark matter (Volkamer and Streicher 1999; Volkamer et al. 1994). According to that theory the Sun is a constant source of dark matter quanta (DMQ) which interact with matter surfaces and can, according to their charge, change the mass of the system they attach to either positively or negatively since they can, in contrast to conventional matter, have both positive and negative mass. Without any eclipse both positive and negative DMQ are irradiated from the Sun towards the Earth. During a solar eclipse positively charged DMQ are focused onto the eclipsed region on Earth whereas the negatively charged DMQ are dispersed (see Fig. 10b). Some experimental evidence to support this hypothesis has been reported (Volkamer 2003). A hitherto unknown interaction between the water/air interface and the DMQ could result in a change in surface tension as observed in this work. It should be pointed out, however, 
that DMQ are not part of the standard model, nor is their existence proven let alone accepted by the scientific community. Nevertheless, they are mentioned here for the sake of completeness.

\section{Majorana Gravitational Shielding}

As a result of some delicate pendulum experiments, Majorana (1919, 1920a, b, c) suggested that gravitational force is weakened when passing through matter. The law which Majorana proposed states that a gravitational ray $l$ of intensity $g$ may be weakened after crossing a layer of material with density $\delta$ according to,

$$
g=g_{0} e^{-h \int \rho d l}
$$

where $h$ is the shielding (or Majorana) coefficient describing the absorption of gravitation by mass. Naturally a syzygy constitutes a situation where such shielding should become effective, resulting in an increased terrestrial gravity in the totality zone of an eclipse. An apparatus measuring surface tension with a microbalance would experience a stronger downwards pull that would read out as increased surface tension, but would actually be due to increased local gravity. Therefore this effect could explain the observations presented in this work. However shortly after Majorana's original publications Russell (1921) showed that calculations of planetary movements based on Majorana's theory did not match with astronomical observations; and suggested that the shielding coefficient could not exceed $1 / 5000$ of the value assigned by Majorana. Since then numerous measurements have been carried out over the years to measure this coefficient, mostly with negative results further reducing the upper threshold of a possible coefficient. An overview is given in a research note by Caputo (2006), a review of conventional explanations of anomalous observations of presumed shielding made during solar eclipses can be found in an arXiv paper by Duif (2004). According to Caputo, research on gravitational shielding based on the use of the Moon or the Earth as screen and using gravimeters the instruments must be one order of magnitude more accurate than the ones used so far. He suggest that the most likely upper limit of the coefficient is the one suggested by Williams et al. (1976), which is

$$
h<2 \times 10^{-21}
$$

Today the existence of gravitational shielding is doubtful at best. Even if it were a real effect, it is many orders of magnitude too small to be measurable with the equipment available for this study.

\section{Heavy Photons}

In Pervozchikov and Sharichin (2005) claimed to have discovered a new kind of electromagnetic radiation emitted by the Sun. Its speed of propagation equals $0.91 c$; and it is supposed to be different from normal ("Maxwellian") radiation. If such particles existed, their impact on the surface of water could possibly disrupt the hydrogen bond network, and thus their blocking by the Moon would result in an increase. Their slower speed would indeed shift the surface tension peak in time, but by a much lower amount, only about $0.2 \mathrm{~s}$. Therefore, in order to account for the much larger (20-40 min) time shift, much longer time constants must be at work. As it is true for DMQ, it should be pointed out that heavy 
photons are not part of the standard model, nor is their existence proven by any other group known to the authors. Nevertheless, they are mentioned here for the sake of completeness.

\section{References}

A.W. Adamson, A.P. Gast, Physical Chemistry of Surfaces, 6th edn. (Wiley, New York, 1967)

M. Allais, Ten Notes published in the Proceedings of the French Academy of Sciences (Comptes Rendus des Seances de l'Academie des Sciences) (1975). Online in English at http://www.allais.info/alltrans/ allaisnot.htm. (in French)

M. Allais, The "Allais Effect" and my experiments with the paraconical pendulum, A memoir prepared for NASA, C-6083 (1999)

E.L. Alpen, Radiation Biophysics (Academic Press, San Diego, 1998)

D.L. Anderson, J. Geophys. Res. 80, 1555-1557 (1975)

C.P. Anil Kumar, R. Gopalsingh, C. Selvaraj, K.U. Nair, H. Johnson Jeyakumar, R. Vishnu, S. Muralidas, N. Balan, J. Geophys. Res. Lett. Atmos. 118, 5098-5104 (2013)

R.H. Backus, R.C. Clark Jun, A.S. Wing, Behaviour of certain marine organisms during the solar eclipse of July 20, 1963. Nature 205, 989-991 (1964)

J.N. Bahcall, M.H. Pinsonneault, Phys. Rev. Lett. 92, 121301 (2004)

J. Barranco, O.G. Miranda, T.I. Rashba, J. High Energy Phys. 2005, 021 (2005)

J. Bartels, N.H. Heck, H.F. Johnston, The three-hour range index measuring geomagnetic activity. Geophys. Res. 44, 411-454 (1939)

G.K. Batchelor, An Introduction To Fluid Dynamics (Cambridge University Press, Cambridge, 1967). ISBN 0-521-66396-2

E. Bayramli, T.G.M. van de Ven, S.G. Mason, Colloids Surf. 3, 131 (1981)

H.A. Bethe, Possible explanation of the solar-neutrino puzzle. Phys. Rev. Lett. 56, 1305-1308 (1986)

S. Bezverkhny, A.L. Osherovich, S.F. Rodionov, Electrophotometric investigation of atmospheric ozone during the solar eclipse. Doklady Ak. Sci. USSR 106, 651-654 (1956)

L. Biermann, Kometenschweife und solare Korpuskularstrahlung. Zeitschrift für Astrophysik 29, 274 (1951)

M. Blumthaler, A. Bais, A. Webb, S. Kazadzis, R. Kift, N. Kouremeti, B. Schallhart, A. Kazantzidis, Variations of solar radiation at the Earth's surface during the total solar eclipse of 29 Mar 2006. Proc. SPIE 6362, 63620F (2006)

R.D. Bojkov, The ozone variations during the solar eclipse of May 20 1966. Tellus 20, 417-421 (1968)

M. Caputo, On new limits of the coefficient of gravitation shielding. J. Astrophys. Astr. 27, 439-441 (2006)

B.W. Carroll, D.A. Ostlie, An Introduction to Modern Astrophysics, 2nd edn. (Benjamin Cummings, San Francisco, 1995), p. 409. ISBN 0-201-54730-9

D.K. Chakrabarty, N.C. Shah, K.V. Pandya, Fluctuation in ozone column over Ahmedabad during the solar eclipse of 24 Oct 1995. Geophys. Res. Lett. 24, 3001 (1997a)

D.K. Chakrabarty, N.C. Shah, K.V. Pandya, Geophys. Res. Lett. 24(23), 3001-3003 (1997b)

Y. Chen, H. Okur, N. Gomopoulos, C. Macias-Romero, P.S. Cremer, P.B. Petersen, G. Tocci, D.M. Wilkins, C. Liang, M. Ceriotti, S. Roke, Sci. Adv. 2, e1501891 (2016)

SNO Collaboration, G.T. Ewan et al., Sudbury Neutrino Observatory Proposal, Report No. SNO-87-12, (1987)

D.W. Deamer, R. Dick, W. Thiemann, M. Shinitzky, Chirality 19, 751-763 (2007)

E. Del Giudice, S. Doglia, M. Milani, G. Vitiello, Nucl. Phys. B 251(13), 375-400 (1985)

D.J. Boersma, L. Gladstone, A. Karle, The IceCube Collaboration, in Proceedings 31st ICRC, Łódź (2009), pp. $1-4$

P.C. Duif, A review of conventional explanations of anomalous observations during solar eclipses, http:// arxiv.org/ftp/gr-qc/papers/0408/0408023.pdf (2004)

I.T. Durham, Rethinking the history of solar wind studies: Eddington's analysis of comet morehouse. Notes Rec. R. Soc. 60, 261-270 (2006)

G. Economou, E.D. Christou, A. Giannakourou, E. Gerasopoulos, D. Georgopoulos, V. Kotoulas, D. Lyra, N. Tsakalis, M. Tzortziou, P. Vahamidis, E. Papathanassiou, A. Karamanos, Eclipse effects on field crops and marine zooplankton: the 29 March 2006 total solar eclipse. Atmos. Chem. Phys. 8, 46654676 (2008)

E.M. Fournier D'Albe, S.I.R. Rasool, Observations de l'ozone atmosphérique pendant une éclipse totale du soleil. Annales de Géophysique 12(1), 72-74 (1956)

E.C. Fuchs, M. Sammer, A.D. Wexler, P. Kuntke, J. Woisetschläger, J. Phys. D Appl. Phys. 49, 125502 (2016) 
E.C. Fuchs, D. Yntema, J. Woisetschläger, J. Phys. D Appl. Phys. (2019). https://doi.org/10.1088/13616463/ab252b. in press

G. Galilei, Cause, Experiment and Science: A Galilean dialogue incorporating a new English translation of Galileo's "Bodies That Stay atop Water, or Move in It" (trans: Stillman Drake). (University of Chicago Press, Chicago, 1612)

J. Geiss, G. Gloeckler, R. Von Steiger, Origin of the solar wind from composition data. Space Sci. Rev. 72, 49-60 (1995)

R.J. Good, Contact angle, wetting, and adhesion: a critical review. J. Adhesion Sci. Technol. 6(12), 12691302 (1992)

S.L. Gray, R.G. Harrison, Diagonsing eclipse-induced wind changes. Proc. R. Soc. A 468, 1839-1850 (2012)

V. Gribov, B. Pontecorvo, Phys. Lett. B 28, 493-496 (1969)

P.K.F. Grieder, Cosmic Rays at Earth (Elsevier, New York, 2001)

R.A. Hayes, J. Ralston, Chem. Aust. 59, 524 (1992)

R.A. Hayes, J. Ralston, Colloids Surf. A 80, 137 (1993a)

R.A. Hayes, J. Ralston, J. Colloid Interface Sci. 159, 429 (1993b)

M. Huang, The Alchemical Imaginary of Homunculi in Fullmetal Alchemist, in Transitions and Dissolving Boundaries in the Fantastic, ed. by C. Lötscher, P. Schrackmann, I. Tomkowiak, A.-A. von Holzen (Lit Verlag GmbH \& Co. KG, Vienna, 2014), p. 46

ICUMSA Methods Book, Bartens (2016), ISBN-10: 3870405562

I.M. Brancus, A. Saftoiu, B. Mitrica, M. Petcu, O. Sima, A. Haungs, G. Toma, M. Duma, A. Bercuci, Correlations between solar events and the cosmic muon flux measured with WILLI detector, in Proceedings 31st ICRC, Łódź (2009), pp. 1-4

N. Jerlov, H. Olsson, W. Schuepp, Measurements of solar radiation at Loevanger in Sweden during the total eclipse 1945. Tellus 6, 44-45 (1954)

D.O. Jordan, J.E. Lane, Aust. J. Chem. 17, 7 (1964)

M.-B. Kallenrode, Space Physics: An Introduction to Plasmas (Springer, Berlin, 2004). ISBN 3-540-20617-5

Y. Kawabata, Spectrographic observation of the amount of ozone at the total solar eclipse of June 19, 1936. J. Astron Geophys. 14, 1-3 (1937). (Tokyo)

A. Kh. Khrigian, G.I. Kuznezov, Some results of the ozone observation during the total solar eclipse of Feb 15, 1961, in Proceedings Third USSR Conference on Atmospheric Ozone (GIMIZ, Leningrad, 1965), pp. $26-32$

L. Kolisko, Die Sonnenfinsternis vom 29. Juni 1927 (Occ. Orient Verlag, Stuttgart, 1927)

T. Kuusela, Effect of the solar eclipse on the period of a torsion pendulum. Phys. Rev. D 43, 2041-2043 (1991)

J.E. Lane, D.O. Jordan, Aust. J. Chem. 23, 2153 (1970)

R. Lewis, Phys. Rev. D 21, 663 (1980)

Luna 1, NASA National Space Science Data Center. Retrieved 2007-08-04

Q. Majorana, Atti della Reale Accademia dei Lincei 28, 160-480 (1919)

Q. Majorana, Philos. Mag. 39, 488 (1920a)

Q. Majorana, Atti della Reale Accademia dei Lincei 29, 23-235 (1920b)

Q. Majorana, On gravitation. Theoretical and experimental researches. Philos. Mag. 39, 488-504 (1920c)

F. Marinho, L. Paulucci, D. Galante, Propagation and energy deposition of cosmic rays' muons on terrestrial environments. Astrobiology 13, 319-323 (2014)

S. Mason, Trends Pharm. Sc. 7, 20-23 (1986)

R. Massoudi, A.D. King, Effect of pressure on the surface tension of water. Adsorption of low molecular weight gases on water at $25^{\circ}$. J. Phys. Chem. 78, 2262-2266 (1974)

G. McHale, S. Aqil, N.J. Shirtcliffe, M.I. Newton, H.Y. Erbil, Analysis of droplet evaporation on a superhydrophobic surface. Langmuir 21, 11053-11060 (2005)

S.P. Mikheyev, AYu. Smirnov, Resonance enhancement of oscillations in matter and solar neutrino spectroscopy. Sov. J. Nucl. Phys. 42, 913-917 (1985)

F.M. Mims, E.R. Mims, Geophys. Res. Lett. 20(5), 367-370 (1993)

Ministry of Defence (NAVY), Admiralty Manual of Navigation, vol 1, 1 (The Stationery Office: London, 1987) p. 277, ISBN 0-11-772880-2

M.M. Weislogel, M.O.J. Azzam, J.A. Mann, Effect of Gravity on Surface Tension, NASA Report (1998), NASA/TM-1998-207915

N. Perevozchikov, V. Sharichin, New kind of electromagnetic radiation, Cellular Neural Networks, Multi-Scroll Chaos and Synchronization, ed. by A. Studenikin, in Proceedings of the 11th Lomonosov Conference on Elementary Particle Physics (World Scientific Publishing Co. Pte. Ltd., 2005) 
M. Narayan, G. Rajasekaran, R. Sinha, C.P. Burgess, Solar neutrinos and the eclipse effect. Phys. Rev. D 60, 073006 (1999)

A.W. Neumann, R. J. Good, Techniques of measuring contact angles. in Surface and Colloid Science, ed. by R.J. Good, R.R. Stromberg (Springer, Boston, MA, 1979)

G. Nicolescu, J. Phys. G Nucl. Part. Phys. 40, 055201 (2013)

I. Pariisky, K. Chen-Chao, Annular sun eclipse of April 19, 1958 on Hainan Island. Acta Geophys. Sinica 10(I), 1-16 (1961)

S.J. Parke, Nonadiabatic level crossing in resonant neutrino oscillations. Phys. Rev. Lett. 57, 1275-1278 (1986)

L.S. Penn, B. Miller, J. Colloid Interface Sci. 77, 574 (1980)

J.L. Pérez-Díaz, M.A. Álvarez-Valenzuela, J.C. García-Prada, The effect of the partial pressure of water vapor on the surface tension of the liquid water-air interface. J. Colloid Interface Sci. 381, 180182 (2012)

A. Pierre Collaboration, J. Abraham et al., Measuerment of the energy spectrum of cosmic rays above 1018 eV using the Pierre Auger Observatory. Phys. Lett. B 685, 239-246 (2010)

B. Pontecorvo, J. Exp. Theor. Phys. (U.S.S.R.) 33, 549-551 (1957)

B. Pontecorvo, J. Exp. Theor. Phys. (U.S.S.R.) 34, 247-249 (1958)

B. Pontecorvo, Sov. Phys. JETP 26, 984-988 (1968)

H.M. Princen, Aust. J. Chem. 23, 1789 (1970)

H.C. Rawlinson, The assyrian canon verified by the record of a solar eclipse, B.C. 763. Athenaeum J. Lit. Sci. Fine Arts 2064, 660-661 (1867)

S.P. Rosen, J.M. Gelb, Mikheyev-Smirnov-Wolfenstein enhancement of oscillations as a possible solution to the solar-neutrino problem. Phys. Rev. D 34, 969-979 (1986)

R.S. Raghavan, in Proceedings of the 25th International Conference on High Energy Physics, Singapore, 1990, ed. by K.K. Phua, Y. Yamaguchi (World Scientific, Singapore, 1991), p. 698

H.N. Russel, On Majorana's theory of gravitation. Astrophys. J. 54, 334-340 (1921)

S. Suess, in Overview and Current Knowledge of the Solar Wind and the Corona, The Solar Probe, NASA/Marshall Space Flight Center. (1999)

S. Santhosh Kumar, R. Rengiayan, Indian J. Tradit. Knowl. 13(4), 716-724 (2014)

B.B. Sauer, W.G. Kampert, J. Colloid Interface Sci. 199, 28 (1998)

E. Saxl, M. Allen, solar eclipse as "seen" by a torsion pendulum. Phys. Rev. D 3(1971), 823-825 (1970)

M. Schütz, P. Schütz, Experimentelle Studien mit der Tropfenbildmethode in Zusammenhang mit einer Sonnen- und Mondfinsternis (Versuchs- und Forschungsanstalt der Stadt Wien, Wien, 1975)

Y. Scolnik, I. Portnaya, U. Cogan, S. Tal, R. Haimovitz, M. Fridkin, A.C. Elitzur, D.W. Deamer, M. Shinitzky, PCCP 8, 333-339 (2006)

J.E. Seebergh, J.C. Berg, Chem. Eng. Sci. 47, 4468 (1992)

A. Shriyan, A.M. Bhat, N. Nayak, Effect of solar eclipse on microbes. J. Pharm. Bioallied Sci. 3, 154157 (2011)

L.B. Slichter, M. Caputo, C.L. Hager, An experiment concerning gravitational shielding. J. Grav. Res. 70, 1541-1551 (1965)

M. Smy, Y. Ashie, S. Fukuda, Y. Fukuda, K. Ishihara, Y. Itow, Y. Koshio, A. Minamino, M. Miura, S. Moriyama, Phys. Rev. D 69, 011104 (2004)

R.S. Steblova, Observations of atmospheric ozone during the eclipse of Feb 15, 1961. Geomagn. Aeron. 2(I), 127-130 (1962). (Moscow)

D. Stranz, Ozone meaurements during solar eclipse. Tellus 13(2), 276-279 (1961)

A. Suzuki, in Physics and Astrophysics of Neutrinos, ed. by M. Fukugita, A. Suzuki (Springer, Tokyo, 1994), p. 414

B. Svensson, Observations on the amount of ozone by Dobson spectrophotometer during the solar eclipse of June 30, 1954. Arkiv för Geofysik 2(28), 494-573 (1968)

Totsuka, Nucl. Phys. B (Proc. Suppl.) 48, 547 (1996)

Values from http://www.finewaters.com/bottled-waters-of-the-world/chile/puyehue. Accessed 17 Oct 2017

Values from Nestle 2017 Water Analysis Report, https://www.nestle-watersna.com/asset-library/Docum ents/AR_ENG.pdf?_ga=2.232512607.1579472576.1509305989-1774703245.1499244240

Values from the label of the water bottle "EDENA eau de source", Société EDENA, 10 rue Eugène Delouise-Rivière des Galets-97419 La Possession La Réunion (2016)

T. van Flandern, X.S. Yang, Allais gravity and pendulum effects during solar eclipses explained. Phys. Rev. D 67, 022002 (2003a)

T. Van Flandern, X.S. Yang, Allais gravity and pendulum effects during solar eclipses explained. Phys. Rev. D 67, $022002(2003 b)$ 
K. Volkamer, Detection of dark-matter-radiation of stars during visible sun eclipses. Nucl. Phys. B (Proc. Suppl.) 124, 117-127 (2003)

K. Volkamer, C. Streicher, Experimental evidence of a new type of quantized matter with quanta as integer multiples of the planck mass. Apeiron 6, 63-82 (1999)

K. Volkamer, C. Streicher, K.G. Walton, J. Fagan, H. Schenkluhn, H. Marlot, Experimental re-examination of the law of conservation of mass in chemical reactions. J. Sci. Explor. 8, 217-250 (1994)

J. Weber, Phys. Rev. C 31, 1468 (1985)

J. Weber, Phys. Rev. D 38, 32 (1988)

L. Wilhelmy, Ann. Phys. Chem. Leipzig 119, 177 (1863)

J.G. Williams, R.H. Dicke, P.L. Bender, C.O. Alley, W.E. Carter, E.G. Currie, D.H. Eckardt, J.E. Faller, W.M. Kaula, J.D. Mulholland, H.H. Plotkin, S.K. Poultney, P.J. Shelus, E.G. Silverberg, W.S. Sinclair, M.A. Slade, D.T. Wilkinson, New test of the equivalence principle from Lunar laser ranging. Phys. Rev. Lett. 36, 551-556 (1976)

Win-Eclipse 3.6, copyright 2002-2007 by Heinz Scsibrany, http://www.wineclipse.net

L. Wolfenstein, Neutrino oscillations in matter. Phys. Rev. D 17, 2369 (1978)

W.-M. Yao et al., Review of particle physics. J. Phys. G Nucl. Part. Phys. 33, 1 (2006)

T. Young, Trans. R. Soc. Lond. 95, 65 (1805)

C.S. Zerefos, D.S. Balis, P. Zanis, C. Meleti, A.F. Bais, K. Tourpali, D. Melas, I. Ziomas, E. Galani, K. Kourtidis, A. Papayannis, Z. Gogosheva, Changes in surface UV solar irradiance and ozone over the Balkans during the eclipse of Aug 11, 1999. Adv. Space Res. 27, 1955-1963 (2001)

Publisher's Note Springer Nature remains neutral with regard to jurisdictional claims in published maps and institutional affiliations. 